Bioacoustics

The International Journal of Animal Sound and its Recording, 1996, Vol 7, pp. 81-117. $0952-4622 / 96 \$ 10$

C 1.996 AB Academic Publishers

$-3+3+3$

\title{
THE MECHANISM OF TUNING OF THE MOLE CRICKET SINGING BURROW
}

\author{
A.G. DAWS ${ }^{*}$, H.C. BENNET-CLARK ${ }^{2}$ AND N.H. FLETCHER ${ }^{3}$ \\ ${ }^{1}$ Department of Zoology, University of Melbourne, Parkville, Vic. 3052, Australia \\ 2Department of Zoology. University of Oxford, South Parks Road, Oxford \\ OX1 3PS, UK \\ ${ }^{3}$ Research School of Physical Sciences and Engineering, Australtan National \\ University, Canberra 0200, Australia
}

\begin{abstract}
1. Experimental and theoretical studies on the acoustics of the singing burrow of the mole cricket Gryllotalpa australis are reported.

2. The burrow typically consists of a bulb about $26 \mathrm{~mm}$ long and $20 \mathrm{~mm}$ in diameter, connected through a constriction of diameter about $10 \mathrm{~mm}$ to a flaring horn with length about $40 \mathrm{~mm}$ and equivalent mouth diameter about $34 \mathrm{~mm}$. The mouth geometry of the burrow differs from one species to another, and the aperture may be either single, double or even multiple. The end of the bulb opposite the horn connects to a narrow exit tunnei of diameter about $8 \mathrm{~mm}$ and length up to $1 \mathrm{~m}$. The singing cricket positions itself close to the constriction between the bulb and the horn and produces a song with a frequency around $2.5 \mathrm{kHz}$.

3. Measurements of sound pressure within the burrow when it is excited by an external sound source at the song frequency show a pressure minimum at the constriction and an amplitude and phase distribution that is consistent with resonance of the burrow at its second modal frequency. The burrow is approximately three-quarters of a wavelength long at this frequency. The same result is obtained when the burrow is excited by a dipole source located near the constriction.

4. Non-parametric model calculations confirm this conclusion and also give broad agreement with the measured response curves over a frequency range from about 1.5 to $5 \mathrm{kHz}$. The calculated curves indicate an additional resonanee at about $1.2 \mathrm{kHz}$ associated with the first mode of the burrow-the Helmholtz or Klipsch resonancewhich is apparently not utilised by the insect. This detail is consistent with earlicr measurements, and is also supported by measured responses reported here that show an increase in sound pressure with decreasing frequency below $2 \mathrm{kHz}$ as predicted by the model.

5 . The measured performance of the burrow is broadly consistent with the model. According to the model, the burrow geometry is close to optimal for maximal sound power radiation.
\end{abstract}

Key words: mole crickets, resonators, sound production, bio-acoustics, burrow, Gryllotalpa.

*The experimental work described here was carried out at Department of Zoology, University of Oxford. 


\section{INTRODUCTION}

Male mole crickets (Orthoptera: Gryllotalpidae) produce loud puretone songs with remarkable efficiency (Bennet-Clark 1970, 1987, Kavanagh 1987). Song is produced from a specially constructed singing burrow (Bennet-Clark 1970, 1987, Nickerson et al. 1979) which has two major components: an outer flared horn and an inner ellipsoidal bulb, joined by a constriction. The insect's wings are placed just outside this constriction during sound production (Figure 1). The exact shape of the horn varies from species to species: Gryllotalpa vinea, a European species, digs a burrow with two openings to the surface (Bennet-Clark 1970), Gryllotalpa australis, an Australian mole cricket, constructs a horn with two, three or four openings to the surface (Kavanagh \& Young 1989), while the burrow of Scapteriscus acletus, an American mole cricket, has only one opening (Nickerson et al. 1979, BennetClark 1987).

While singing, the mole cricket stands in the constriction between the horn and bulb, with its head and prothorax in the bulb and its abdomen in the throat of the horn (Bennet-Clark 1970, Kavanagh \& Young 1989). Its forewings are raised across the burrow on the horn side of the constriction so that they extend from side to side of the burrow; the plane at which the insect's wings are placed has been termed the burrow datum (Bennet-Clark 1987). The forewings act as a dipole sound source (Olson 1957, Bennet-Clark 1971) between the two components of the burrow. Destructive acoustic interference that would otherwise occur between the two surfaces of the forewings is partially prevented by the close fit between the raised wings and the sides of the burrow.

Previous work on the singing burrow of Scapteriscus acletus showed evidence that it behaved as a tuned system; the free field response of a dipole or doublet sound source increased by $24 \mathrm{~dB}$ when it was placed within the singing burrow in such a way as to mimic the action of the insect's wings (Bennet-Clark 1970, 1987). This gain in sound pressure was limited to a narrow frequency band, centring on the carrier frequency of the species' calling song, about $2.7 \mathrm{kHz}$ (Bennet-Clark 1987). The tonal purity of this tuning, or its Quality factor or Q, was measured at about 3. This suggested that the horn acts as a tuned acoustic transformer which allows the relatively small wings of the male to produce low frequencies at high intensities. It also suggested that the tuned burrow provided a resistive load on the insect's wings at the throat of the horn (Bennet-Clark 1987) which allowed the insect to produce a pure tone song. If the burrow were untuned or were tuned to a different frequency from that of the insect's wings, it would be likely to provide a substantial reactive loading that would affect the wings' resonant frequency and thus might alter the song frequency; there is some evidence that this may occur (Bennet- 


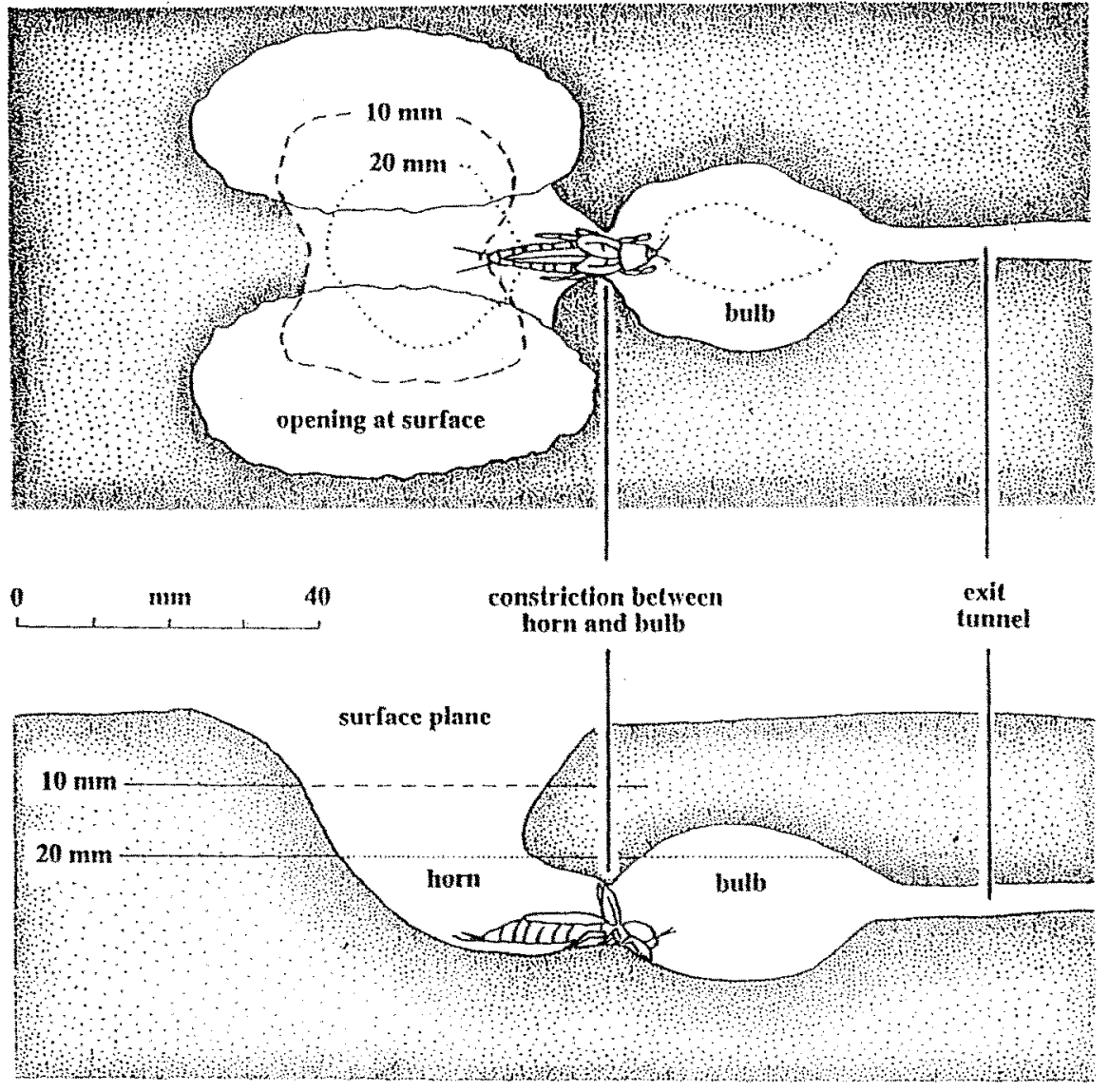

Figure 1. Plan and vertical section scale drawings of a typical singing burrow of Gryllotalpa australis, to show the position of the singing insect and its position relative to the horn, bulb and constriction.

Clark 1987). However, these experiments did not explore the mechanism by which the tuning was achieved.

We now report on experiments with plastic model burrows that are rigid replicas of the singing burrow of an Australian mole cricket Gryllotalpa australis and thus allow examination of the acoustics of different parts of the burrow. These experiments have led to a substantial reinterpretation of the acoustics of mole cricket singing burrows. 


\section{MATERIALS AND METHODS}

\section{Polyester casts of mole cricket burrow}

Plaster casts were made of the burrows of mole crickets (Gryllotalpa australis) singing in gardens around the grounds of the University of Melbourne between February and April of 1991. Plasticine replicas of these burrows were constructed by hand using detailed comparative measurement and embedded in clear polyester casting resin. Care was taken to limit the amount of catalyst used so as to restrict the heat produced by the setting process to levels below the melting point of the plasticine. The resultant blocks measured $0.15 \mathrm{~m}$ long $\times 0.10 \mathrm{~m}$ wide $\times 0.05 \mathrm{~m}$ deep. After the resin had cured fully, the plasticine was removed using a steam-cleaner and detergents. The five model burrows used in the experiments reported here were typical of the natural range of burrow shapes.

The plane of the insect's wings when in the natural singing position is about $3 \mathrm{~mm}$ on the horn side of the constriction between the horn and the bulb. Where necessary, a hole was drilled into the interior of the burrow to allow a probe microphone to be placed in the burrow at the constriction; when not in use, this hole was blocked with a metal rod. When measuring sound pressure levels in the horn, the microphone diaphragm was placed $10 \mathrm{~mm}$ below the mouth of the horn. For measurements in the bulb, the microphone was inserted through the exit tunnel with the remaining space packed with cotton wool, with the microphone diaphragm halfway along the length of the bulb.

Three model mole crickets were made to simulate the presence of the singing animal. These were made of Blu-Tack (a pliable, re-useable plastic adhesive) mounted on $0.5 \mathrm{~mm}$ diameter wire. The first of these approximated to the dimensions of a male cricket, being $25 \mathrm{~mm}$ long and $8 \mathrm{~mm}$ diameter. The second was $40 \mathrm{~mm}$ long and $10 \mathrm{~mm}$ diameter, and the third was $20 \mathrm{~mm}$ long and $5 \mathrm{~mm}$ diameter.

\section{Experimental enclosure}

Experiments were carried out within an $0.3 \mathrm{~m} \times 0.3 \mathrm{~m} \times 0.5 \mathrm{~m}$ enclosure made of Ilform acoustic anechoic foam of $85 \mathrm{~mm}$ thickness. Hard surfaces, such as those of the resin blocks themselves and those of the support rods or micromanipulators, were covered with a $20 \mathrm{~mm}$ thick layer of cotton wool to reduce their effect upon measurements made. Support rods used had diameters of less than $12.5 \mathrm{~mm}$, and clamps used to hold microphones were less than $25 \mathrm{~mm}$ across. Previous tests made within this enclosure showed that echoes were 30 $40 \mathrm{~dB}$ below the signals being measured (Bennet-Clark and Young 1992). 


\section{Sound production chain}

The model burrows were driven during the course of the experiments using the sound production chain shown in Figure 2. Tone bursts or steady-state signals were produced by a Tektronix Function Generator FG501a which could be gated by a Tektronix Pulse Generator PG501. This signal was passed through a centre-tapped audio-frequency transformer, to give two antiphase voltages which were then fed into the left and right inputs of a Marantz PM 230 stereo amplifier. The live side of the outputs of the two amplifiers were used to drive the Electret Doublet Source (see below) thus doubling the available voltage output from the power amplifier. The frequency of the signal was monitored using a Testlab M2365 multimeter which had previously been tested for accuracy against a Tektronix DC 503 universal counter.

\section{Sound sources}

The signal was used to drive either a purpose-built Electret Doublet Source (E.D.S.), as described in Bennet-Clark (1987), or a Sony earphone that had been supplied with a WM R50 Recording Walkman. The $10 \mathrm{~mm}$ diameter E.D.S. was fixed to a $200 \mathrm{~mm}$ long brass strip

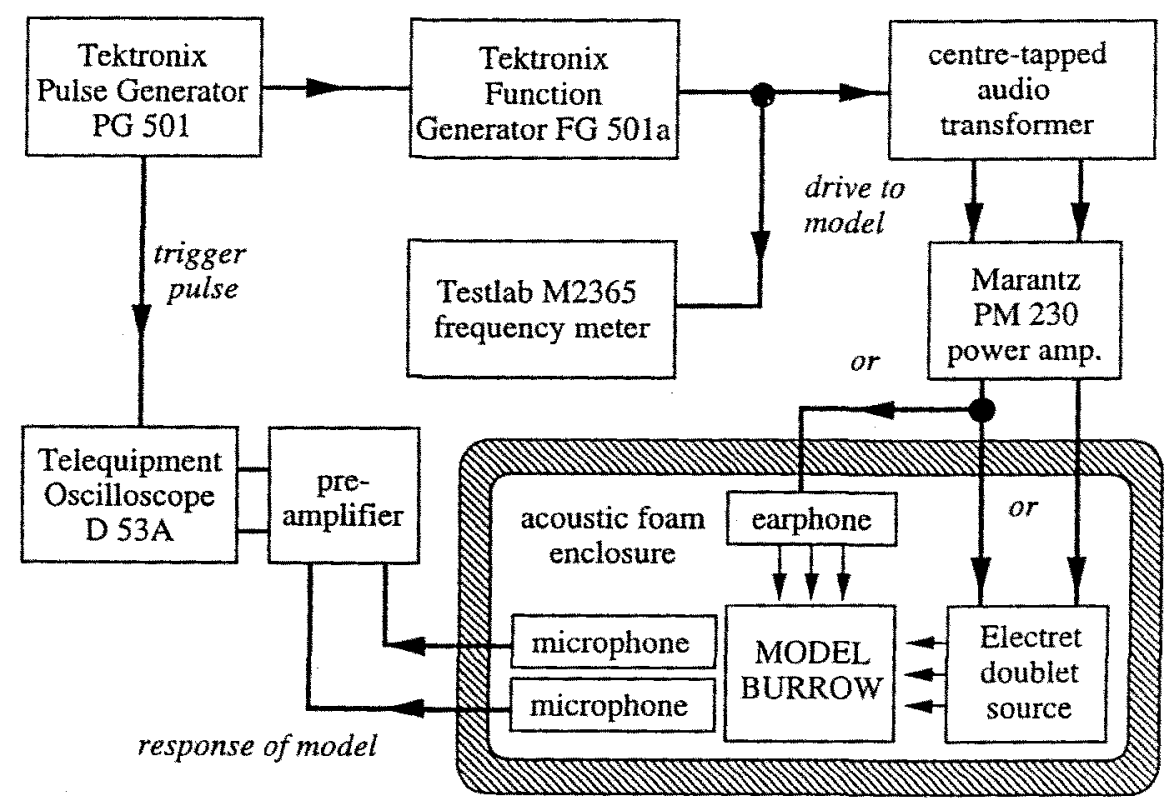

Figure 2. Block diagram of the apparatus used to generate sound pulses to drive plastic models of mole cricket singing burrows and to measure their acoustics. 
upon which a scale had been glued. The voltage drive to the Electret Doublet Source was $70 \mathrm{~V}$ peak to peak. The E.D.S. could be manipulated up and down the burrows to provide an internal acoustic dipole or doublet source drive. The E.D.S. response has weak resonance at about $17.5 \mathrm{kHz}$.

A $17.5 \mathrm{~mm}$ diameter Sony earphone was used as an external drive to the burrows. On test, mounted as a loudspeaker at a range of $50 \mathrm{~mm}$ from a Tandy microphone, it was found that the sound output rose steadily from $1 \mathrm{kHz}$ to $14 \mathrm{kHz}$ but showed no resonances with $\mathrm{Q}$ values exceeding 1 . This relatively resonance-free response was confirmed by driving the earphone with a 5 cycle tone burst, which it reproduced as a pulse with a rectangular envelope that built up and decayed within half a cycle. The earphone was fixed to a horizontal support rod with Blu-Tack $120 \mathrm{~mm}$ above the midline of the model burrow, vertically above the two openings of the horn.

\section{Sound measurements}

Two pairs of microphones were used to measure the response of the model burrows. The first pair of microphones were constructed using $7 \mathrm{~mm}$ diameter Realistic Electret Tie Pin Microphones (catalogue 331063), from the same manufacture batch, fixed to a $150 \mathrm{~mm}$ length of flexible lead rod of $4.5 \mathrm{~mm}$ diameter. These microphones have a weak resonance at about $15 \mathrm{kHz}$ but the response between 1 and $10 \mathrm{kHz}$ is smooth and free of resonances, and their response to tone bursts in this frequency band is reproduced cleanly. When placed $0.2 \mathrm{~m}$ from the earphone in anechoic conditions with their front faces co-planar, these microphones gave matched responses within 5 per cent. or $\pm 0.5 \mathrm{~dB}$ and with less than $5 \mu$ s time difference between them over all frequencies between $1 \mathrm{kHz}$ and $10 \mathrm{kHz}$.

The second pair of microphones were constructed using another pair, from a different manufacturing batch, of the same type of Realistic Electret microphone unit modified to hold a $5 \mathrm{~mm}$ long probe tube of $1.25 \mathrm{~mm}$ diameter (Figure 3). This was fixed onto an aluminium support rod of $6 \mathrm{~mm}$ diameter, upon which a scale had been fixed.

Resonances in the probe microphones' frequency response were damped by pushing tufts of phosphor bronze wool into their probe tubes until, by trial and error, the response was non-resonant and closely similar. After these adjustments, these microphones also gave matched responses within $\pm 0.5 \mathrm{~dB}$ and below $5 \mu$ s time difference from $1 \mathrm{kHz}$ to $10 \mathrm{kHz}$.

The probe tube internal diameter was $0.8 \mathrm{~mm}$. This means that, since the resonances of the probe tube are damped, the acoustic impedance of the probe tube is of order $10^{9} \mathrm{SI}$ acoustic ohms. As this is considerably higher than that of the horn (about $10^{7} \mathrm{ohms}$ ) the probe microphone has negligible disturbing effect. The small size of the probe 


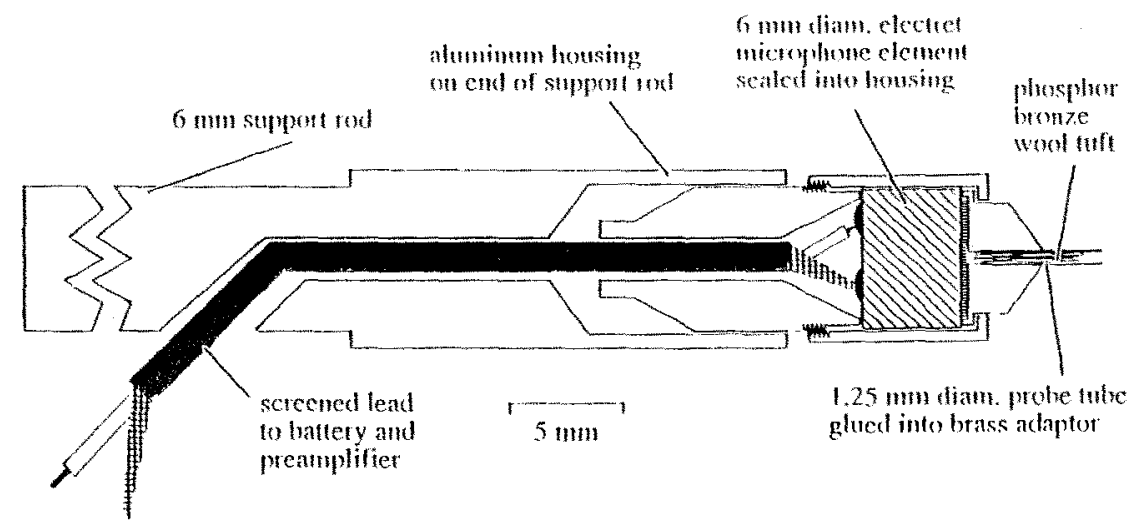

Figure 3. Diagram of the construction of one of a pair of probe microphones used to make comparative measurements at different parts of the same model burrow.

allows its position to be specified to within about $0.5 \mathrm{~mm}$.

A fifth microphone was also used. It was made from a $6 \mathrm{~mm}$ diameter electret microphone element (from Henry's Radio, Edgware Road, London). The microphone element was glued into a $7 \mathrm{~mm}$ outside diameter aluminium alloy housing, into the end of which was sealed a probe tube made from a $5 \mathrm{~mm}$ long section of $1.25 \mathrm{~mm}$ outside diameter stainless steel tubing from a hypodermic needle. The microphone and its housing were glued onto $100 \mathrm{~mm}$ long $6 \mathrm{~mm}$ diameter rod to fit onto a micromanipulator. The response of this microphone was also adjusted using a tuft of phosphor bronze wire wool.

The voltage outputs of the microphones were fed into a Telequipment D 53 A oscilloscope via a purpose built preamplifier. Voltages from comparisons between paired microphones or between treatments were measured on the screen and converted into relative sound pressure level $\mathrm{L}$ in decibels $(\mathrm{dB})$ using the equation

$$
L=20 \log _{10}\left(V_{1} / V_{2}\right)
$$

where $V_{1}$ and $V_{2}$ are the voltages measured.

Each microphone was calibrated to both the Electret Doublet Source and the earphone in free field, anechoic conditions. For the purpose of calibration the E.D.S. was placed in a baffle made of cardboard, which was $0.2 \mathrm{~m} \times 0.3 \mathrm{~m}$, to represent its action within the burrow. Paired microphones were placed $0.2 \mathrm{~m}$ from the sound source, which was driven over a frequency range of $1 \mathrm{kHz}$ to $10 \mathrm{kHz}$. Probe microphones were placed with the tips of the probe tubes touching and equidistant from the source. Unmodified electret microphone units were placed side by side with their front faces coplanar. The voltage 
output of the microphones under these conditions was converted to $\mathrm{dBV}$ (decibels relative to 1 volt) and taken as a baseline response for that sound source. This was then subtracted from subsequent burrow responses, after correcting for the distance between the source and microphones during different manipulations. The difference thus provided a measure of the pressure gain between the measuring position in the burrow and this particular standardised source configuration, for which the acoustic volume flow can be calculated..

No absolute calibration of these microphones was made because they were always used to make comparisons between sound pressures in two places or two experimental manipulations. On occasions when more than two microphones were used at once, the respective pairs of microphones were calibrated to one another over the range from $1 \mathrm{kHz}$ to $10 \mathrm{kHz}$.

\section{Measurement of resonant frequency and $Q$}

The resonant frequency was determined by direct observation from the oscilloscope in three ways. First, the resonant frequency was that at which the maximum amplitude of response to a $2 \mathrm{~ms}$ tone burst with a square envelope was attained. Second, the frequency at which the sound pulse had the slowest rate of decay after the end of the driving tone burst was measured. Third, when the free field response of the sound source was subtracted from the response of the model burrow, the frequency at which the resultant gain was maximal was taken as the resonant frequency of the burrow. Phase differences between two signals were also measured directly from the oscilloscope screen, taking into account any time delay between microphones such as that produced by a probe tube.

$Q$, the quality factor of a resonator, is a measure of the sharpness of tuning of the resonator and ropresents the relative width of the resonance curve $3 \mathrm{~dB}$ below the maximum (this is sometimes known as the $Q_{3 \mathrm{~dB}}$ to distinguish it from other criteria). The effective gain in the amplitude of the response at resonance is $Q$ times the driving amplitude.

The most reliable estimate of $Q$ for resonators of the type used here is obtained by calculating the natural logarithm of the ratio of the amplitudes of successive cycles of the free decay (the logarithmic decrement $\delta$ ) following a driven oscillation. The $Q$ value is then

$$
Q=\pi / \delta
$$

This method has been used here. The repeatability of successive measurements of $Q$ was better than 5 per cent. For a more detailed 
treatment of the measurement of $\mathrm{Q}$ in this context and its implications see Bennet-Clark and Young (1992).

\section{RESULTS}

\section{Burrow dimensions}

The dimensions of ten plaster casts of burrows were measured and used to calculate mean values. Effective diameters were derived from cross-sectional areas at different positions within the burrow. The axial length of the horn was determined using a flexible wire rod of $2 \mathrm{~mm}$ diameter with a scale attached. The wire rod was bent into a curve to match the curvature of each individual horn, and then inserted such that it ran along the approximate axial centre of the horn (Figure 1). The distance along this axis from the centre of the constriction to the surface plane was then read from the scale. The mean axial horn length was $40 \mathrm{~mm}$ (s.d. $7 \mathrm{~mm}, \mathrm{n}=10$ ), and its effective diameter increased from $11 \mathrm{~mm}$ (s.d. $1 \mathrm{~mm}, \mathrm{n}=10$ ) at the throat to $34 \mathrm{~mm}$ (s.d. $3 \mathrm{~mm}, \mathrm{n}=10$.) at the mouth. The bulb had a mean length of $26 \mathrm{~mm}$ (s.d. $2 \mathrm{~mm}, \mathrm{n}=10$ ), and a mean diameter of $20 \mathrm{~mm}$ (s.d. $2.5 \mathrm{~mm}, \mathrm{n}=$ 10). The exit tunnel had a mean diameter of $10 \mathrm{~mm}$ (s.d. $1 \mathrm{~mm}, \mathrm{n}=10$ ).

\section{Sound pressure levels in an internally driven burrow}

Sound pressure levels were measured simultaneously in the bulb and at the mouth of the horn while the burrow was driven internally by the Electret Doublet Source over a range of frequencies and at several points within the burrow (Figure 4). Both the bulb and the horn showed a resonant peak with a mean $Q$ value of 6.7 (s.d. 1.4, $n=5$ ) at a mean frequency of $3.0 \mathrm{kHz}$ (s.d. $0.3 \mathrm{kHz}, \mathrm{n}=5$ ). At this burrow resonant frequency, the pressures within the bulb and the horn were closely similar, and were approximately $180^{\circ}$ out of phase.

When the Electret Doublet Source was moved $5 \mathrm{~mm}$ on either side of the constriction, either deeper into the bulb or outwards into the horn, the pressure response in both the horn and the bulb dropped, while still exhibiting the $3.0 \mathrm{kHz}$ resonance. A $10 \mathrm{~mm}$ displacement of the Electret Doublet Source either way further reduced the pressure response (Figure 4).

Considerably below the burrow resonance, at about $1.2 \mathrm{kHz}$, a substantial resonance was clearly audible. However, it has not been possible to measure the effective burrow gain below this frequency because the free field output of the Electret Doublet Source below 2 $\mathrm{kHz}$ is very low. 

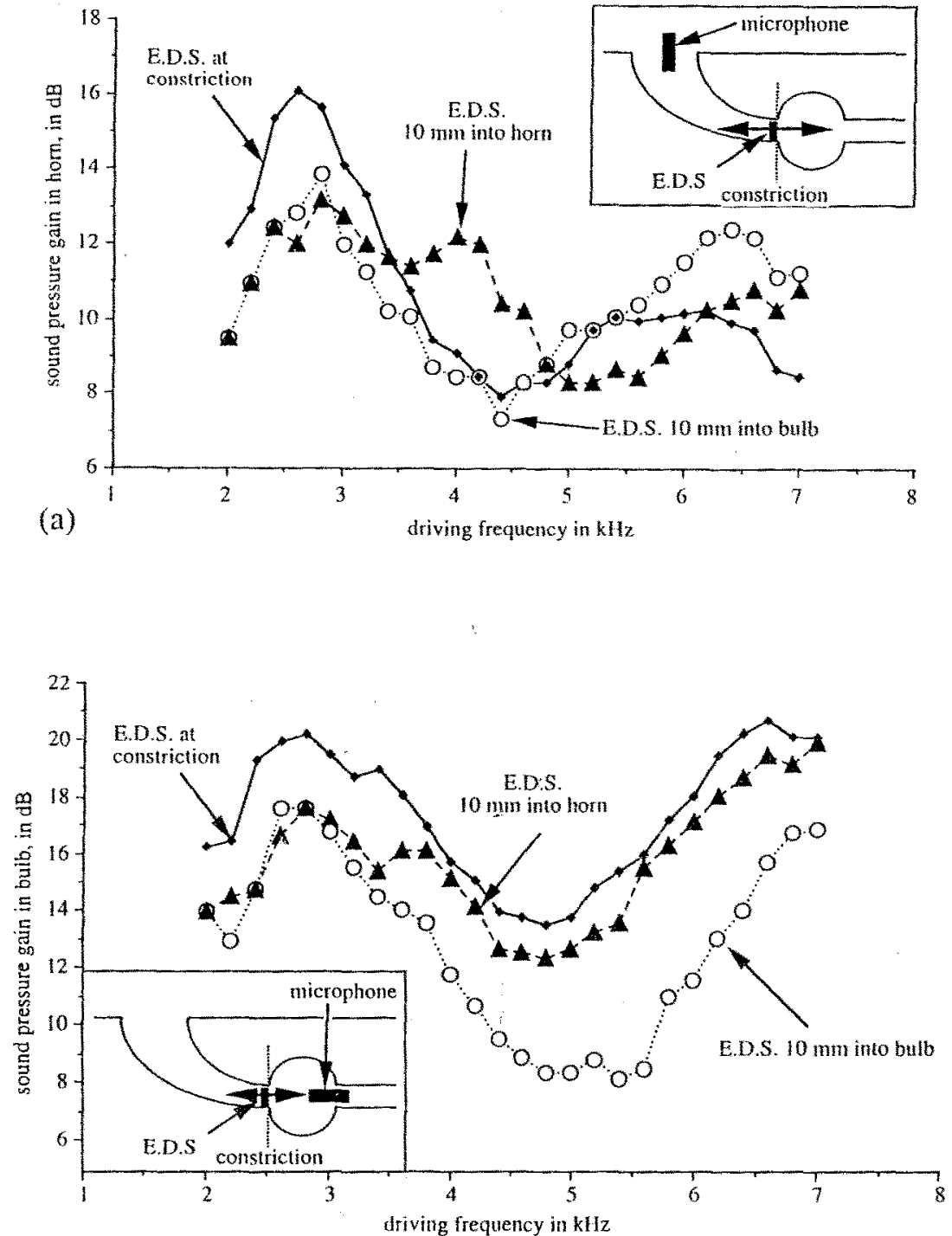

(b)

Figure 4. Sound prossures, expressed as gain in decibels, against driving frequency in $\mathrm{kHz}$, measured in a model burrow driven by the Electret Doublet Source (E.D.S.) in one of three positions, at the constriction, at $10 \mathrm{~mm}$ into the horn or at $10 \mathrm{~mm}$ into the bulb. The insets show the driving and measuring conditions. (a) Sound pressures measured with a microphone placed $10 \mathrm{~mm}$ inside the mouth of the horn. (b) Sound pressures measured with a microphone inserted through the exit tunnel to the middle of the bulb. 


\section{Response of the burrow to an external sound source}

Sound pressure levels were measured simultaneously in the bulb, at the mouth of the horn, and close to the constriction while the burrow was driven externally over a range of frequencies (Figure 5a).

The $3.0 \mathrm{kHz}$ resonant peak was evident in both the horn and the bulb. However, the sound pressure level at the constriction was about $14 \mathrm{~dB}$ lower than those in the horn and bulb at this frequency. Below the resonant frequency the pressure at the constriction approximately equalled that in the bulb, while above resonance it matched that in the horn. At resonance, the pressure responses of the horn and the bulb were out of phase, with the sound pressure in the bulb lagging approximately $180^{\circ}$ behind that in the horn.

The results of a previous unpublished study (Daws, 1991. Hons. Thesis, University of Melbourne) show a resonant peak at about 1.2 $\mathrm{kHz}$, a second at $3 \mathrm{kHz}$, and a third that fell between $5.0 \mathrm{kHz}$ and 6.5 $\mathrm{kHz}$ (Figure 5b). In this study, the model burrow was driven externally via the short exit tunnel using a Sony earphone. The burrow response was measured using a Bruel \& Kjaer sound pressure level meter type 2230 with an octave filter set type 1624 and a $1 / 2$ inch microphone type 4155 placed $1.0 \mathrm{~m}$ from the burrow mouth, within a $1.5 \mathrm{~m} \times 1.0 \mathrm{~m} \times$ $0.8 \mathrm{~m}$ enclosure of Sonex acoustic anechoic foam $85 \mathrm{~mm}$ thick. The free field response of the Sony earphone, measured under the same conditions, was subtracted from the burrow response to give the approximate gain in $\mathrm{dB}$ provided by the burrow.

\section{The effect of the mole cricket on the system}

Model mole crickets were placed in the natural singing position within the burrow. The sound pressure response of the horn to an external drive over a range of frequencies is shown in Figure 6.

In each burrow, the addition of the model cricket increased the sound pressure in the horn by 2 to $4 \mathrm{~dB}$ at and below the resonant frequency; the effect varied slightly with the size of the model cricket used (Figure 6). Above resonance the presence of the cricket had little effect on the acoustics of the burrow. Hence the gain of the burrows at $2.5 \mathrm{kHz}$, the carrier frequency of the calling song (Kavanagh 1987), is some $2 \mathrm{~dB}$ greater when the presence of the insect is taken into account than is suggested by the $3.0 \mathrm{kHz}$ resonant peak obtained from empty burrows. 


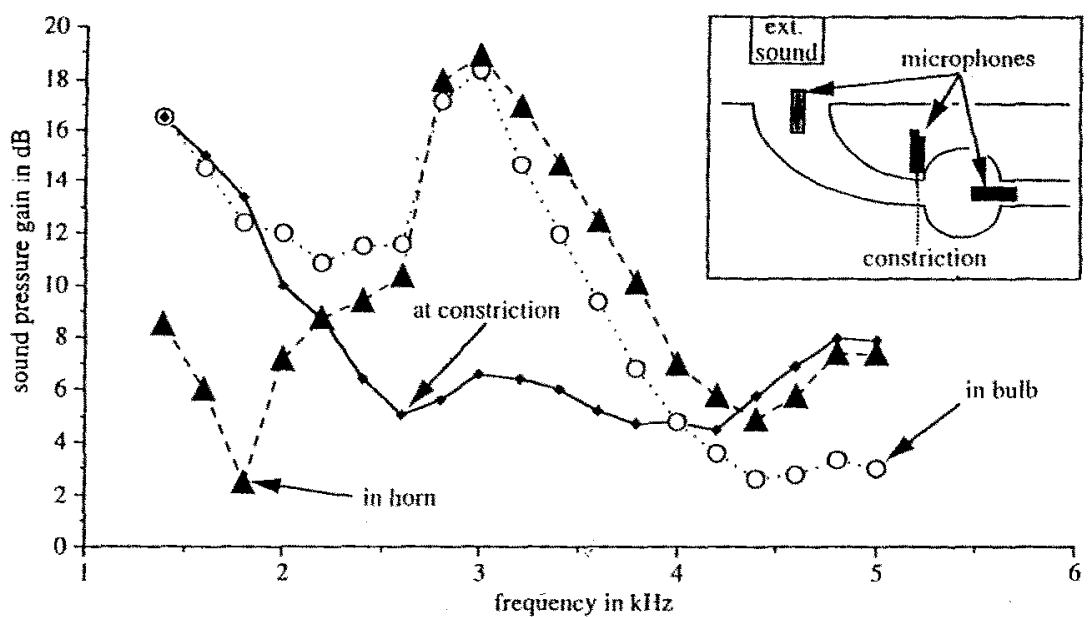

(a)

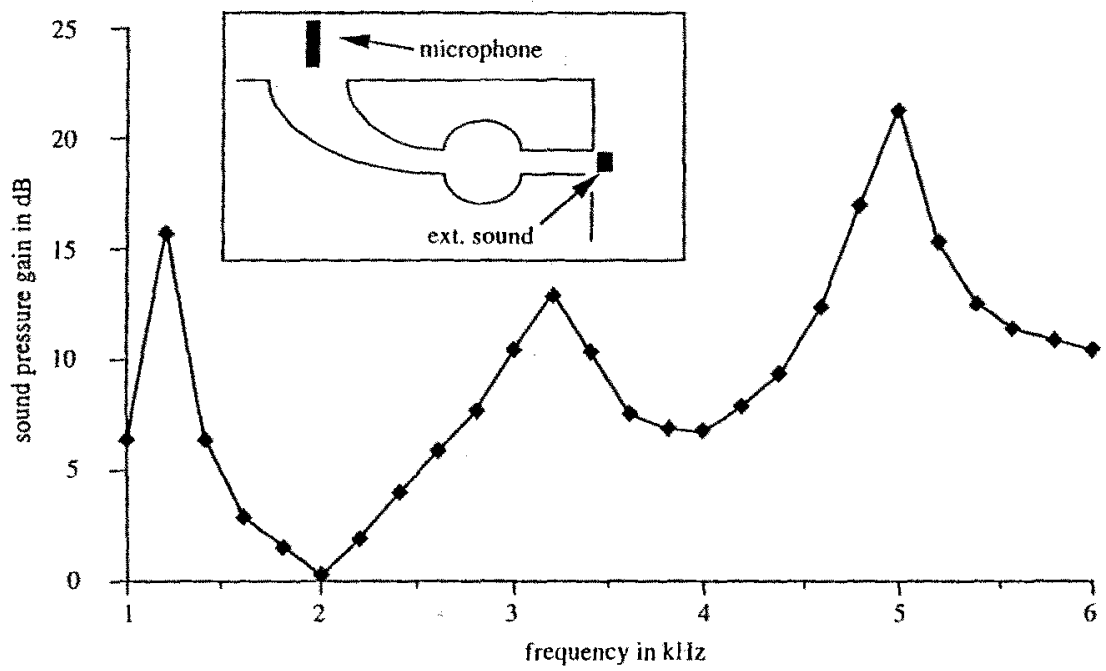

(b)

Figure 5. Sound pressures, expressed as gain in decibels, against driving frequency in $\mathrm{kHz}$, measured in a model burrow when driven by an external sound source. The insets show the driving and measuring conditions. (a) Measurements were made simultaneously at three positions: at the constriction; at $10 \mathrm{~mm}$ inside the mouth of the horn; and at the middle of the bulb. (b) Sound pressure levels measured with a microphone placed $1.0 \mathrm{~m}$ from the mouth of the horn while the horn was driven via the exit tunnel. 


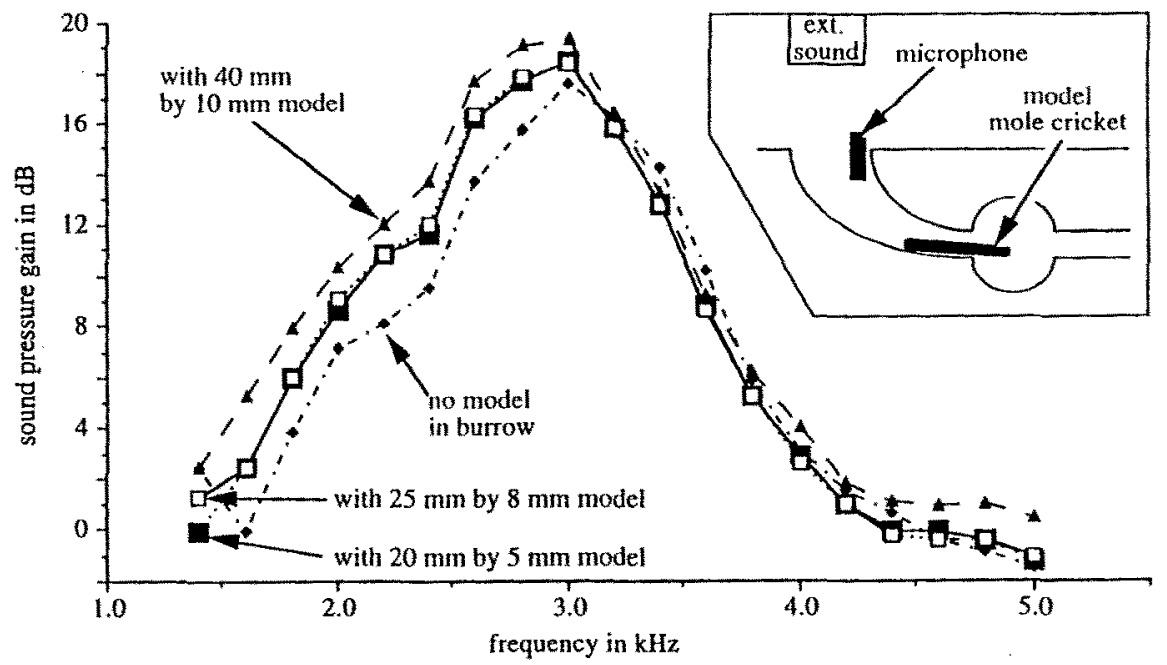

Figure 6. Sound pressures, expressed as gain in decibels, measured in a model burrow when driven by an external sound source, either without a model mole cricket or with a $20 \times 5 \mathrm{~mm}$ model, a $25 \times 8 \mathrm{~mm}$ model (similar in size to the insect) or a $40 \times 10 \mathrm{~mm}$ model placed in the position occupied by the singing insect. Measurements were made $10 \mathrm{~mm}$ inside the mouth of the horn. The inset shows the driving and measuring conditions.

\section{Pressure and phase differences at different points within the burrow}

Sound pressures along the length of the burrow were measured using a probe microphone inserted via the exit tunnel while the burrow was externally driven at its resonant frequency (Figure 7a). The sound pressure in the bulb decreased markedly as the constriction was approached, reaching a null point at or around the constriction. The sound pressure then increased as the microphone was moved a further $10 \mathrm{~mm}$ towards the mouth of the horn; further measurements made from the mouth of the horn showed that about $10 \mathrm{~mm}$ outwards from the datum the sound pressure remained more or less constant to within about $10 \mathrm{~mm}$ of the horn mouth at the surface plane. The sound pressure at the constriction of different burrows was between $12 \mathrm{~dB}$ and $20 \mathrm{~dB}$ below that in either the bulb or the horn.

Change in relative phase angle along the length of the burrow was measured using two microphones (Figure $7 \mathrm{~b}$ ). One microphone was placed at the mouth of the burrow to act as a reference, and the second was inserted via the exit tunnel as described above. The pressure response of the bulb lagged about $180^{\circ}$ in phase behind that measured at the burrow mouth. As the microphone passed through the 

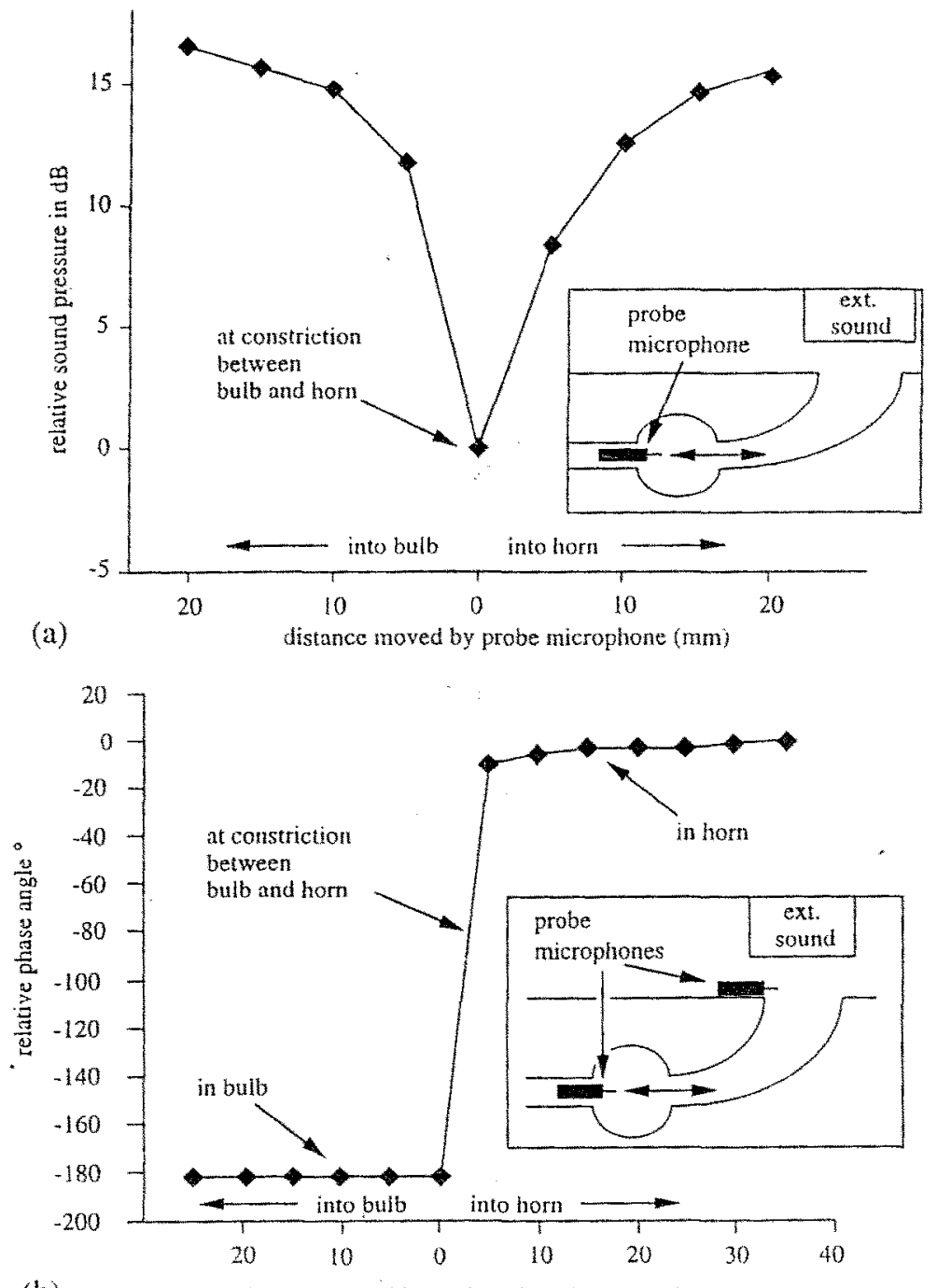

(b)

distance moved by probe microphone ( $\mathrm{mm}$ )

Figure 7. Plots of microphone response against measurement position. The burrow was driven at its resonant frequency by an external sound source, and the measuring microphone was moved in $5 \mathrm{~mm}$ steps from the bulb into the horn (see inset). (a) The relative sound pressure within the burrow, expressed in $\mathrm{dB}$ relative to the voltage measured at the constriction. Note that there is a sound pressure null at or close to the constriction. (b) The phase of the driving sound was determined by a second microphone placed at the mouth of the horn. Note that the phase changes abruptly by approximately $180^{\circ}$ as the measuring microphone is moved through the constriction. 


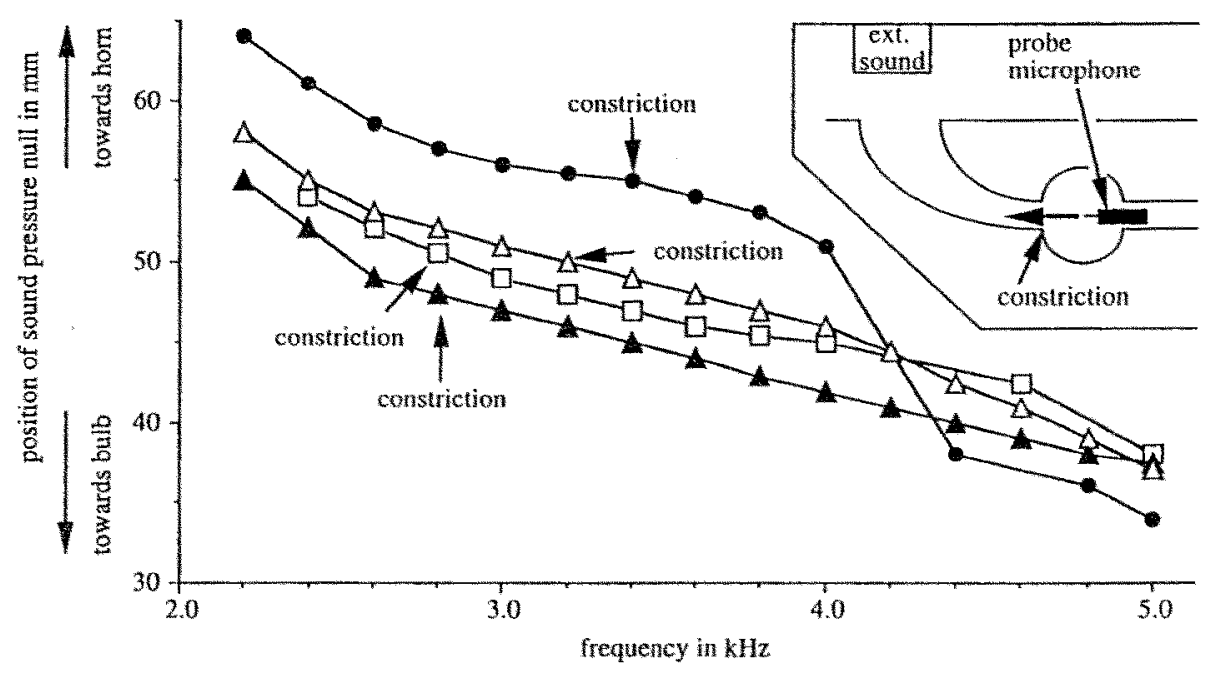

Figure 8. Plots of position of the sound pressure null against the frequency at which the null occurred for four model burrows. The burrows were driven by an external sound source at $0.2 \mathrm{kHz}$ intervals between $2.0 \mathrm{kHz}$ and $5.0 \mathrm{kHz}$, and the null was located with a probe microphone with a scale attached (Figure 3) moved from the bulb into the horn. Arrows show the measurement made at the constriction which corresponds with the resonant frequency of each burrow.

pressure null at the constriction the phase reversed, such that the pressure response in the horn was approximately in phase with that at the burrow mouth.

The position of this local pressure null and plane of phase transition altered with the frequency of the driving source. Figure 8 shows the position of the null determined over a range of frequencies from $2 \mathrm{kHz}$ to $5 \mathrm{kHz}$ in $0.2 \mathrm{kHz}$ intervals for four model burrows. At frequencies below the burrow resonance, the null was located in the throat of the horn. At the resonant frequency of the individual burrow (about $3.0 \mathrm{kHz}$ ) the null was situated approximately at the constriction, and at higher frequencies it moved into the bulb.

\section{DISCUSSION}

The burrow as a resonant system

The results presented here support the hypothesis that the singing burrow of mole crickets acts as a single, closely coupled resonant system (Bennet-Clark 1987). However, the mechanism by which this resonance is achieved differs considerably from that previously 
reported. The singing burrow of the mole cricket has been described as a form of Klipsch horn (Bennet-Clark 1970, 1987). The design of this short horn includes an air chamber behind the diaphragm to offset the throat inductance (Klipsch 1941). The bulb of the mole cricket burrow was thought to fulfil a similar function.

This description of the burrow is closely analogous to a longnecked Helmholtz resonator, with the complication that the neck is no longer a simple tube, but rather a flared horn. The properties of such a resonator have been well examined elsewhere (Fletcher 1992, Bennet-Clark and Young 1992). The lowest mode is the Helmholtz resonance, the frequency of which is determined by the volume of the bulb and the length and cross-section of the neck. It is this mode that is made use of by Klipsch in his short horn design (Klipsch 1941), with the relationship between the cavity and horn described by the equation

$$
V=2.9 A R
$$

where $V$ is the volume of the bulb in cubic millimetres, $A$ is the throat area in square millimetres, and $R$ is the axial distance in which this cross-sectional area doubles in millimetres. In this mode the acoustic pressure has the same phase throughout the whole resonator and its magnitude increases smoothly from a small value at the open mouth to a uniform maximum in the bulb. Clearly this is not the more associated with the resonance near $3.0 \mathrm{kHz}$ identified in our measurements.

The next mode of such a resonator has a standing wave with, approximately a half-wavelength along the neck, a pressure null and consequent $180^{\circ}$ phase change near the junction of the neck and bulb and a pressure maximum at the remote wall of the bulb. The frequency of this mode is determined by the linear dimensions of the bulb and tube or horn. This behaviour agrees adequately with our measurements. There exist also many higher modes with more complex phase behaviour, but these are progressively more highly damped by the exit tunnel. This tunnel, because of its length (up to 2 $m$, A.G.D. pers. obs.), its relatively small diameter, and its rough and porous walls, can be expected to act as a simple acoustic resistance contributing to the damping of the system but having little effect on its resonance frequencies, as we verify in the model calculations detailed below.

The dimensions of the burrow support the notion of a standing wave at $3.0 \mathrm{kHz}$. This is in agreement with the model calculations below, which show that the average dimensions of the mole cricket burrow place its first resonance at about $1.2 \mathrm{kHz}$ and its second resonance at about $3.0 \mathrm{kHz}$. The effective length of the horn, when corrected for the radiation impedance of an opening in a baffle, is somewhat longer than the axial length of $40 \mathrm{~mm}$. This is close to $1 / 2$ 
a wavelength at $3.0 \mathrm{kHz}$. The bulb length of $26 \mathrm{~mm}$ is about $1 / 4$ of a wavelength at $3.0 \mathrm{kHz}$. Thus the bulb appears to be acting as a tuned cavity that extends the length of the horn. When the insect is in the singing position with its wings placed in the constriction plane, sound directed into the bulb will be reflected back towards the horn. The dimensions of the bulb ensure that when the reflected wave reaches the throat of the horn it is in phase with the sound pressure within the horn. This constructive interference may help to negate the loading effect of the throat impedance of the horn upon the vibrating wings of the cricket.

The position of the null plane, or pressure node of the standing wave, is determined by the wavelength of the driving frequency (Figure 8). At higher frequencies the $1 / 4$ wavelength distance is decreased, and the null plane is found further within the bulb. At lower frequencies the 1/4 wavelength distance is correspondingly greater, and the null plane lies beyond the constriction, within the throat of the horn. By building a bulb that is approximately $1 / 4$ of a wavelength long at the resonant frequency of the burrow, the mole cricket can position the null plane to fall at or near the constriction between the bulb and the throat of the horn.

This 1/4 wavelength relationship was first commented upon by Nickerson et al. (1979). However, the bulb lengths in some of the species studied by Nickerson et al. (1979) and Bennet-Clark (1970, 1987) fall closer to $1 / 3$ of a wavelength. This discrepancy may possibly be explained in terms of the effective length of the bulb, which will differ somewhat from the actual length due to its ellipsoid, rather than cylindrical shape.

The standing wave model is further supported by the experimental manipulations of the Electret Dipole Source within the burrow. The most efficient transduction of electrical power to acoustic power occurred at the resonant frequency of the burrow when the dipole sound source was placed at the constriction (Figure $4 \mathrm{a}, \mathrm{b}$ ). The model burrows provided between $12 \mathrm{~dB}$ and $20 \mathrm{~dB}$ gain to a dipole source placed in the natural singing position of the insect. If there is a standing wave within the burrow, with a pressure node at the constriction, then this would be the most effective position to place a dipole source. At this point the pressure on either side of the source is minimal and the particle velocity should be at a maximum.

The phase relationships on either side of the pressure null alsc support a standing wave model. The $180^{\circ}$ phase shift at the pressurt null is typical of a standing wave (Gough et al. 1983). Similarly, the constant phase along the length of the horn and within the bulb show that the sound pressures in the burrow are not due to a progressive wave, which would cycle through approximately $270^{\circ}$ of phase alon the length of the burrow.

The measured resonant frequency of $3.0 \mathrm{kHz}$ is consistentl: 
higher than the $2.5 \mathrm{kHz}$, carrier frequency of the calling song but may, in part, be because the acoustic properties of the plastic resin burrows differ somewhat from those of the earth in which the animal digs its singing burrow. The presence of the insect in the singing position appears to modify the response of the burrow to give extra gain at frequencies around $2.5 \mathrm{kHz}$ (Figure 5). The $\mathrm{Q}$ value of about 6 implies that the plastic model burrows are more sharply tuned than the burrows of Scapteriscus acletus constructed in soil where a $Q$ of about 3 was measured (Bennet-Clark 1987); this difference is hardly surprising as the porous walls of natural burrows will damp the natural resonance more than the reflective walls of the plastic models.

The horn acts, in part, as an impedance matching device. The area of the wave front increases as it propagates along the horn from the source. If a sound source is less than about one third of the sound wavelength in diameter, its source impedance per unit area is less than that of the surrounding fluid medium (Olson 1957), so it will not radiate sound efficiently. Thus the increase in area from the insect's wings to the burrow mouth is central to the ability of the horn to produce low frequency sound from a small source. This is consistent with the use to which similar horns are put in acoustical engineering (Olson 1957).

\section{Tuning the burrow}

The dimensions of the burrow play a large role in determining the resonant frequency of the burrow. However, other factors do modify the tuning of the burrow. The presence of the insect decreases the horn throat area, so the horn will be tuned as if the rate of flare of the horn was increased. This will have an effect on the propagation of low frequencies within the burrow. The size of the cricket relative to the dimensions of the burrow will also effect the amount of acoustic flow that occurs from one side of the insect's wings to the other. When the fit between the wings and the walls of the burrow at the constriction is close, acoustic "short circuiting" at the edges of the wings in minimised. As individual mole crickets vary in size, the dimensions of the horn and bulb of each burrow must not only match each other, but also suit the dimensions of the particular cricket digging the burrow. Burrow construction appears to be a trial and error process (BennetClark 1987). It involves several phases of remodelling while the burrow is being built, presumably with adjustment to the size of the bulb.

More precise tuning may be obtained by adjusting the exact position of the insect's wings when singing. On the evidence presented here, the wings will be most effective as a sound source when they lie directly in the constriction: However, the position of the sound pressure null in the burrow moves in and out with the driving 
frequency. Thus, for a given frequency there will be a particular plane at which the pressure will be at a local null and the throat of the horn will offer a resistive load to the insect's wings. Given the natural variation in frequency of the calling song, this allows for a certain latitude in the tuning of each burrow. A mole cricket might then position itself so that its wings lie in the optimum plane to correspond with the frequency of vibration of its wings.

The American mole cricket Scapteriscus acletus moves inwards or outwards along the throat of the horn between or during the bursts of song that it produces during the initial tuning-up phases of the evening chorus (Bennet-Clark 1987). This may represent an attempt to find the point at which the pressure on either side of its forewings is balanced or at which the burrow is tuned to the resonant frequency of its wings. Thus, it seems the tuning of the burrow takes place in two phases. During construction, the dimensions of the horn and the bulb are matched to each other and to the size of the insect. Then, after the burrow is completed, the insect chooses the optimum position within the burrow from which to sing, such that the acoustic load on either side of its forewings is matched.

\section{MODEL CALCULATIONS}

To support the explanation for the acoustics of the cricket burrow outlined above, it is very helpful to have a physical model that is sufficiently realistic that it represents a good approximation to the real situation but is at the same time sufficiently simple that it is possible to calculate its acoustic behaviour in precise quantitative terms without undue computational labour. This approach to the analysis of acoustic systems in biology has been discussed in detail by Fletcher (1992), following a briefer treatment by Fletcher and Thwaites (1979). Because of the simplifications made in constructing the model, we should not expect exact agreement between its predictions and the results of measurements on the real system, but the general behaviour should be reproduced and the quantitative predictions should be good approximations to the measurements.

It is important to emphasise that such a model contains no arbitrarily adjustable parameters and is not simply a way of representing the experimental data. Rather, it is an independent test of the validity of the hypotheses underlying the interpretation of that data. It differs greatly, therefore, from the more familiar parametric models in which generalised differential equations representing the behaviour of a system are written down, and the unknown numerical parameters involved are chosen to give agreement with experimental data. It would certainly be possible to include adjustable parameters in the model we present here in order to improve its agreement with 
100

experiment, but this is not in accord with its purpose.

\section{Setting up the Model}

In setting up this model, we must compromise between simplicity and accuracy. We know, for example that the behaviour of a curved horn which is very difficult to calculate, is nearly the same as that of a straight horn with the same axial length and cross-sectional behaviour (Keefe and Benade 1983), and this is easy to calculate. We therefore replace the real curved horn by a straight horn in our model, and we take the flare to be simply exponential, because this is a good approximation which makes the calculation easy. Similarly, we replace the egg-shaped cavity at the end of the burrow by a straight cylindrical pipe of similar length and average diameter, while the exit tunnel is taken to be of effectively infinite length, an approximation justified by its large physical length and the attenuating nature of its walls. The model for the burrow is thus as shown in Figure 9(a). For the twoaperture burrow studied here we should, perhaps, treat it as consisting of two identical horns in parallel, but as we note later this complication is unnecessary.

As described previously, measurements of three different kinds were made. In the first measurements, the results of which are shown in Figure 4, the burrow was excited by a doublet source located near the datum position, in order to simulate the natural singing situation for the cricket. The diagram of Figure $9(\mathrm{~b})$ shows the acoustic flows in the model calculation for this situation. In the second set of measurements, as shown in Figure 5(a) and Figure 7, the burrow was excited by an external sound source placed outside the open horn mouth. Figure 9(c) shows the model for this case. Finally, the burrow was excited by a sound source placed outside the truncated exit burrow, as in Figure 5(b), and the model for this is shown in Figure $9(\mathrm{~d})$. In each case, the radiation impedance at the open end of the burrow, shown as a hatched area, includes terms both for the end correction and for the radiation resistance, each of which depends upon frequency.

The doublet source representing the cricket or the doublet electrostatic source is shown in detail in Figure $9(\mathrm{~b})$. In the case of the E.D.S., we know that the membrane is a few micrometres in thickness and has a resonance frequency of about $10 \mathrm{kHz}$. Calculation shows that the membrane impedance at $3 \mathrm{kHz}$ is several times the characteristic impedance for an air-filled pipe of the same diameter, so that it is a reasonable approximation to treat it as an acoustic flow source $U_{0}$ which is in parallel with an annular gap that allows for a flow $U_{2}-U_{0}^{0}$ in the space between the source and the walls of the horn throat. The dimensions of this shunt can be estimated from the known geometry of 


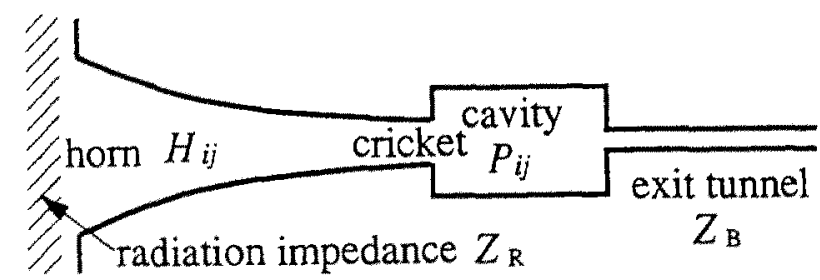

(a)

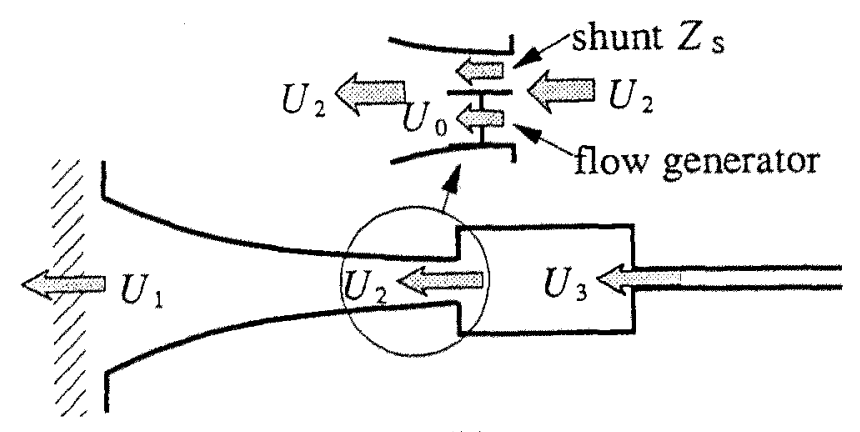

(b)

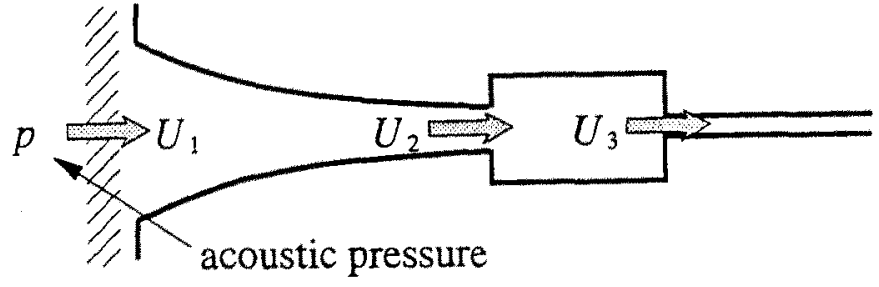

(c)

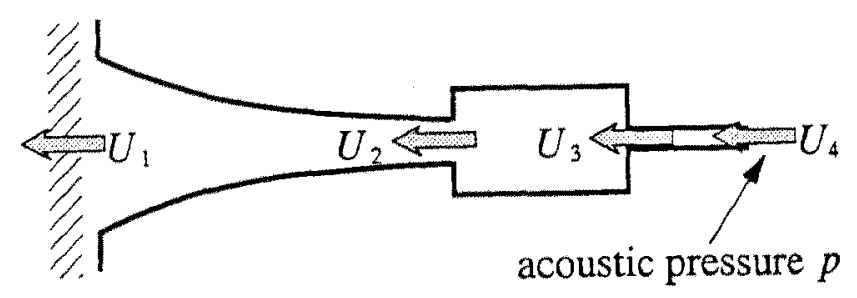

(d)

Figure 9. (a) Idealised model of the cricket burrow. The horn is straightened, the cavity is taken to be a short cylindrical pipe, and the exit tunnel is taken to be of infinite length. The symbols represent the acoustic impedances of the elements of the system.

(b) Acoustic flows $U_{i}$ under internal excitation of the burrow by the cricket or the E.D.S. flow source. Details of flows near the source are shown as an inset. (c) Acoustic flows when the burrow is excited by an acoustic pressure source outside its mouth. (d) Acoustic flows when the external source is outside the truncated exit tunnel. 
the transducer. We adopt a similar model for the wings of the insect, though this is complicated by the fact that the operating frequency is close to the resonance frequency of the wing panels.

The acoustic output from the singing burrow is represented by the flow $U_{1}$ into the resistive part of the radiation impedance at the horn mouth. In the case of external excitation, as in (c), sound reaches the mouth of the horn from an incident sound wave, and this is represented by a simple pressure source $p$. The pressure measured by a microphone placed at various parts of the system can be calculated when we know the acoustic flows and impedances involved.

\section{Details of the calculation}

It is not necessary to foliow the details of the calculation in order to understand the results. Since these details are important, however, they are documented in the Appendix. Application of the method, which is standard in physical acoustics, to biological systems is discussed by Fletcher and Thwaites (1979) and, more particularly, by Fletcher (1992). Here we simply outline the steps leading to the final results.

The acoustic properties of the various components of the system are represented by acoustic impedances, which are generally frequency-dependent complex quantities. For components that are short compared with the sound wavelength, the flow in at one side is equal to the flow out at the other, and the acoustic impedance is a single quantity which we have denoted by $Z_{\mathrm{R}}$ for the radiation impedance and $Z_{\mathrm{S}}$ for the impedance of the shunt space around the cricket body or the doublet source. The exit tunnel also requires only a single impedance quantity $Z_{B}$ because it is blocked at its far end. In contrast, the flows at the two ends of the horn and the cavity are not necessarily equal, because they are comparable in length with the sound wavelength, and there may be appreciable compression of the air inside them. They are therefore each represented by a matrix of four impedance coefficients which we have denoted by $H_{i j}$ and $P_{i j}$ for the horn and cavity pipe respectively. Details of the way in which the numerical values of these impedance quantities are determined by the geometry of the elements they describe are given in the appendix.

In each of the two experimental situations there are three acoustic flows $U_{1}, U_{2}$ and $U_{3}$ that we would like to determine. In the case of internal excitation, these are all proportional to the acoustic flow $U_{0}$ generated by the wings of the cricket or by the dipole source, while in the case of external excitation they are proportional to the sound pressure $p$ in the incident sound wave. Analysis of the model vields, in each case, a set of three simple algebraic equations from which these flows can be determined, as shown in equations (A1) and 
(A5) of the appendix. We can solve this set of equations in each case and write down an explicit algebraic result for each of the flows, but the expressions are complicated and do not convey much meaning until they have been evaluated numerically and plotted.

Of particular interest is the acoustic power $\Pi$ radiated from the mouth of the burrow. This energy is lost from the system and appears in the model as the power dissipated in the resistive part $R_{\mathrm{R}}$ of the radiation impedance $Z_{\mathrm{R}}$. Its magnitude is given as

$$
\Pi=R_{R} U_{i}^{2}
$$

if $U_{1}$ is a r.m.s. value, and to make this meaningful we must express the flow $U_{1}$ out of the mouth of the horn in terms of the flow $U_{0}$ created by the cricket's wings. Examination of the way in which this radiated power $\Pi$ varies as we change the geometry of the burrow allows us to see how well the cricket optimises its design.

\section{Calculated results}

The calculations outlined above and in the Appendix can be easily followed through in numerical form, once the physical dimension of the burrow have been specified, and give results that can be compared directly with experiment. The values assumed for the dimensions are given in Table 1. The effective length of the horn has been taken to be about 10 percent greater than its geometrical length to the ground plane to allow for wavefront curvature at its mouth, which is justifiable on theoretical grounds. The effective diameter of the cavity is taken to be that cylindrical diameter that gives the actual cavity volume. Since the cavity is approximately ellipsoidal, this diameter is approximately $(213)^{1 / 2} \approx 0.82$ times the transverse ellipsoidal diameter, which we take to be the average measured for the natural burrows.

The easiest case to calculate is that in which the burrow is

TABLE I

Assumed geometrical parameters

\begin{tabular}{lr} 
Length of horn (including constriction) & $45 \mathrm{~mm}$ \\
Effective mouth diameter of horn & $34 \mathrm{~mm}$ \\
Throat diameter of horn (constriction) & $12 \mathrm{~mm}$ \\
Length of electret dipole source & $3 \mathrm{~mm}$ \\
Diameter of electret dipole source & $10 \mathrm{~mm}$ \\
Length of cavity & $26 \mathrm{~mm}$ \\
Effective diameter of cavity & $17 \mathrm{~mm}$ \\
Diameter of exit tumel & $7 \mathrm{~mm}$ \\
\hline
\end{tabular}


excited by an external sound source; as in Figure 9(c). Figure 10 shows the frequency variation of the calculated sound pressure levels $10 \mathrm{~mm}$ inside the mouth of the horn, at the centre of the bulb, and in the constriction, for this case. The reference pressure in this case is simply that measured at a distance from the source equal to the distance to the horn mouth. By comparison with the corresponding curves of Figure 5(a), it is readily seen that the shapes of the calculated curves are in good agreement with experiment over the range $1.5-5 \mathrm{kHz}$, and that the absolute values of the pressure gains are also quite well represented. The calculated sharp minimum in the horn pressure near $4.5 \mathrm{kHz}$ is not present in the measured curves, but this disagreement is not significant, because such a minimum would almost inevitably be masked by stray sound.

The inset in Figure 10(a) shows the rise towards a resonance near $1 \mathrm{kH} \%$ is suggested in the experimental curves, though they do not extend to a low enough frequency to define it. The $Q$ factor of the major resonance is determined, in the model, by damping due to radiation and to sound absorbed in the long burrow tunnel. The calculated value is about 5, which is close to the value 6 measured on the artificial burrow. The exact shape of the minimum in the pressure curve in the constriction depends rather sensitively upon the length of the cavity, which is what we should expect, and is related to the sensitivity to exact location of the microphone or of the singing cricket, as shown in Figures 4 and 7 .

In the model, the long exit burrow is treated as an infinite pipe, which is justified by the fact that its length is much greater than the sound wavelength and its walls have appreciable absorption. Numerical experiments in which the value assigned to the burrow impedance is varied show that this has essentially no effect on the position of the system resonances but only affects their sharpness-a narrow burrow reduces the acoustic losses and makes the resonance peaks sharper. The burrow contributes rather more than half the losses in the model, since wall losses in the horn and cavity are neglected and the only other loss is by radiation from the horn mouth.

It is also simple to calculate the situation explored in Figure 5(b), in which the burrow is excited by a sound source placed outside the exit burrow, which was truncated for this purpose to a length of about $30 \mathrm{~mm}$. The calculational model is shown in Figure $9(\mathrm{~d})$ and the calculated result in Figure 10(b). Once again the shape of the curve is in excellent agreement with experiment. The absolute value of the gain could not be calculated because of uncertainties in the records of the experiment.

Turning now to the more difficult problem of internal excitation by the electrostatic doublet source, Figure 11 shows the frequency variation of the sound pressure level at a position $10 \mathrm{~mm}$ inside the mouth of the horn and also at the centre of the bulb calculated from 

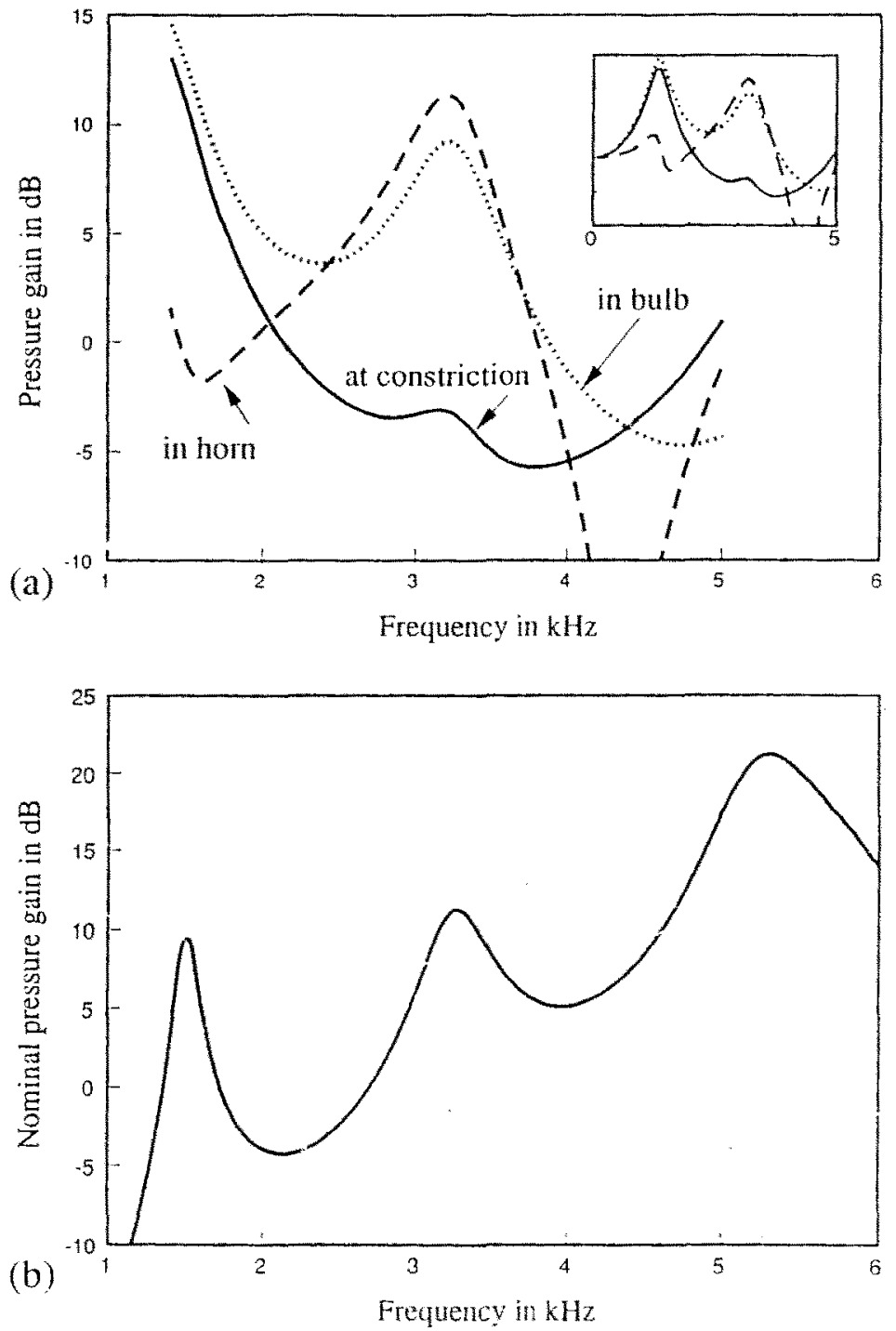

Figure 10. (a) Calculated pressure gain, relative to an incident plane wave, as measured $10 \mathrm{~mm}$ into the mouth of the horn (dashed curve), in the centre of the bulb (dotted curve) and at the datum position in the constriction (full curve). These curves should be compared with the measured data of Figure 5(a), in which the three measurement positions are indicated similarly. The inset shows the calculated response over the full frequency range $0-5 \mathrm{kHz}$ and includes the resonance near $1.2 \mathrm{kHz}$. (b) Calculated pressure outside the burrow mouth when it is excited by a pressure source placed outside the truncated exit burrow. This curve should be compared with the measured data in Figure 5(b). 


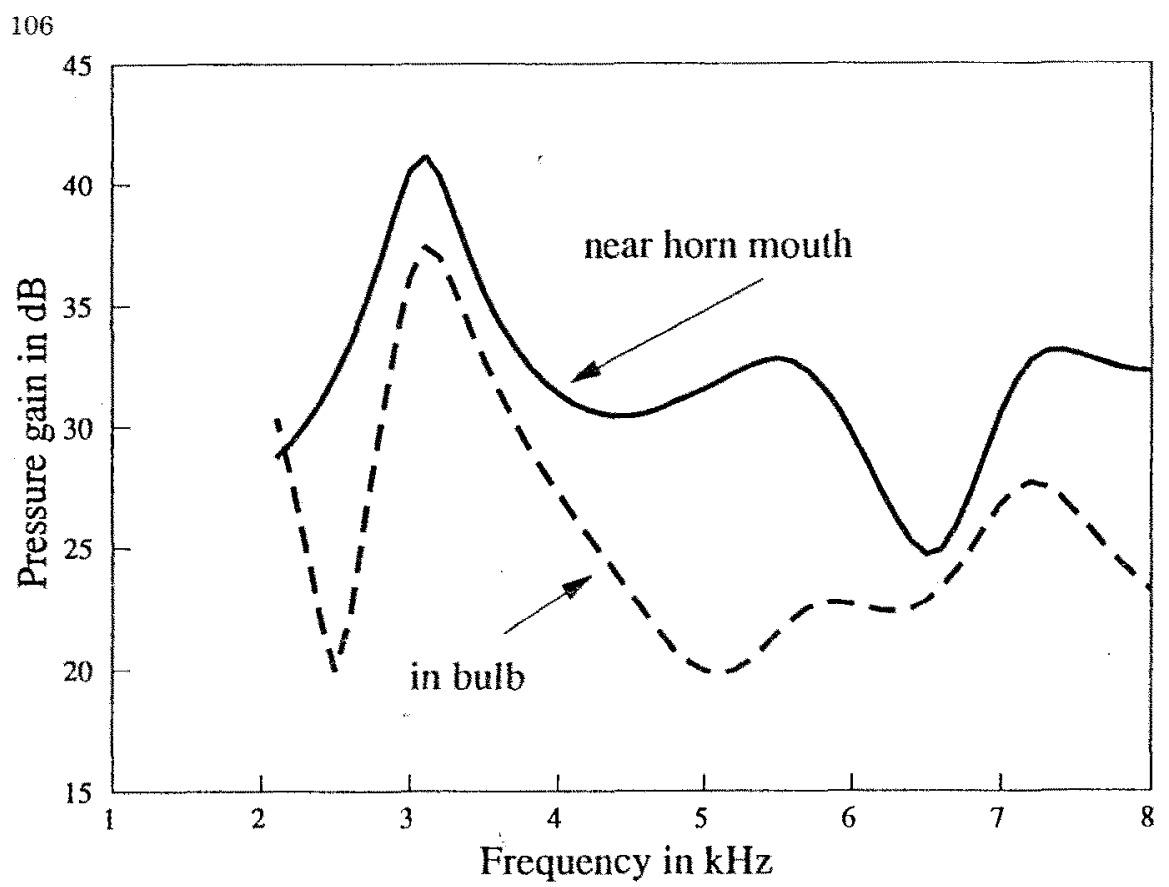

Figure 11. Calculated pressure gain (relative to the baffled E.D.S. at a distance of $200 \mathrm{~mm}$ ) at points $10 \mathrm{~mm}$ into the mouth of the horn and in the centre of the bulb. These curves should be compared with the measured data, shown as full lines in Figures $4(\mathrm{a})$ and $(\mathrm{b})$.

the model of Figure 9(b). For comparison with the experimental results, the calculated sound pressure levels have been expressed relative to the sound pressure level $p_{0}$ produced by the doublet source, mounted in a cardboard baffle, at a distance of $200 \mathrm{~mm}$, as in the calibration procedure. If the acoustic flow produced by the source is $U_{0}$, then the pressure $p_{0}$ produced at a distance $r$ on the axis by the baffled source at frequency $f$ is given by Morse $(1948$, pp. 326-328) in a form that can be simplified to

$$
p_{0}=\rho f U_{0} / r
$$

where $\rho$ is the density of air. When we calculate the difference in decibels between the calculated pressure $p$ and the reference pressure $p_{0}$, the flow quantity $U_{0}$ simply cancels.

The calculated curve for the pressure just inside the horn mouth shows a sharp maximum pressure gain at a frequency near $3 \mathrm{kHz}$, with subsidiary maxima near 5.5 and $7 \mathrm{kHz}$. These results are in acceptably good qualitative accord with the experimental measurements shown as a solid line in Figure 4(a) for the E.D.S. at the constriction, or by a 
dashed line for the E.D.S, slightly into the horn, over the range 2-7 $\mathrm{kHz}$. The calculated value of the gain is, however, higher than that measured by about $20 \mathrm{~dB}$, and the peaks are much sharper. This suggests that the quantitative disagreement is partially due to the omission of wall damping and other losses from the simplified model, though the main problem is perhaps the inaccurate modelling of the dipole source.

The calculated pressure in the bulb is also shown in Figure 11, and again agrees well in frequency dependence with the measured behaviour shown in Figure 4(b) for the range of positions of the E.D.S. investigated. There is a main peak in the calculated response near $3 \mathrm{kHz}$ and a subsidiary peak near $7 \mathrm{kHz}$, while in the measurements these two peaks are of nearly equal height. Again there is a quantitative discrepancy of about $15 \mathrm{~dB}$, which we can ascribe to the same modelling problems.

\section{Phase relations}

The lumped-parameter method of solution adopted for the model is not well adapted to calculating the variation of response with position near the datum constriction, as reported theoretically in Figure 7, because the model treats each part of the burrow as a unit. It is easily possible, however, to calculate the phase difference between the pressure in the bulb and in the horn. This calculation shows that the relative phase is close to zero for the resonance near $1.2 \mathrm{kHz}$ (the actual zero phase frequency is $1.3 \mathrm{kHz}$ ) and close to $180^{\circ}$ for the resonance near $3 \mathrm{kHz}$ (the actual zero phase frequency is $3.1 \mathrm{kHz}$ ).

As well as supporting the measurements reported in Figure 7(b), this analysis confirms our interpretation of the nature of the two burrow resonances. The resonance near $1.2 \mathrm{kHz}$, which has approximately constant phase throughout the whole burrow, is the first mode of the system, which can be thought of either as a Helmholtz resonance of the cavity and horn or equivalently as the first "quarterwavelength" resonance of a tube stopped at the buried end. In each case the frequency is modified from the simplistic value because of the non-ideal shape of the burrow (Fletcher 1992, p. 183-186). The second resonance is most easily thought of as the second resonance of a stopped tube or stopped horn, which is then about three-quarters of a wavelength long, though again the frequency is modified by the nonideal shape. In this mode there is a $180^{\circ}$ phase change at a distance about one-third of the way from the stopped end, and the phases in the two sections are approximately constant. In each case the phase constancy is only approximate because of the resistive load contributed by the exit burrow at the closed end. 
108

\section{Optimisation of geometry}

From the model of Figure 9(b) and equation (4), it is also possible to calculate the radiated acoustic power, which is the quantity of real physical and biological interest, and to see how this varies with the parameters describing the geometry of the burrow. If we assume a song frequency of $3 \mathrm{kHz}$, corresponding approximately to the resonance peak measured in Figures 4-6 and calculated in Figures 10-11, then these functional dependences are as shown in Figure 12 for the major burrow parameters, the other parameters being kept at the values given in Table 1. The diameter of the exit tunnel can also be varied; the effect is not large, but radiated power is greater and the $1.2 \mathrm{kHz}$ peak more prominent when the tunnel is narrow. Although the parameters are somewhat interdependent, it can be seen that a cricket singing at about $3 \mathrm{kHz}$ comes close to optimising the geometry of the burrow to produce maximum radiated sound power, although a horn about $5 \mathrm{~mm}$ longer would appear to be even more effective. The fact that the optimal burrow mouth diameter appears to be smaller than that actually used is connected with frequency matching rather than with radiation efficiency, since changing the mouth diameter also changes the horn resonance frequency.

\section{Frequency anomaly}

This overall result is what we should expect from an evolutionary perspective, but we are now faced with the puzzling fact that the actual song frequency of the cricket is in the range 2.3 to $2.8 \mathrm{kHz}$, with a mean value of $2.5 \mathrm{kHz}$, rather than being about $3 \mathrm{kHz}$ or a little higher as we would expect from this optimisation. This discrepancy does not appear to be the result of any oversimplification in our model for the burrow, since the calculated response agrees tolerably well with that measured, both for external excitation and with the internal electret source.

We can immediately rule out environmental conditions such as temperature and humidity in the natural environment, since these differ insufficiently from the laboratory situation to reduce the sound velocity appreciably. We note, however, that the resin model burrows in our experiments, and also the theoretical model, treat the walls as being smooth and rigid, and it is possible that this does not represent adequately the real earth-walled burrow. Using a transmission line analogy (Morse 1948, p. 254) the sound velocity $v$ in a tube of radius $a$ and cross-section $S=\pi a^{2}$ filled with air of density $\rho$ can be calculated from the acoustic inertance $L=\rho / S$ per unit length and the acoustic compliance $C=S / \rho c^{2}$ per unit length, where $c$ is the sound velocity in free air. The resulting expression is $v=(L C)^{-1 / 2}$, from which we find 

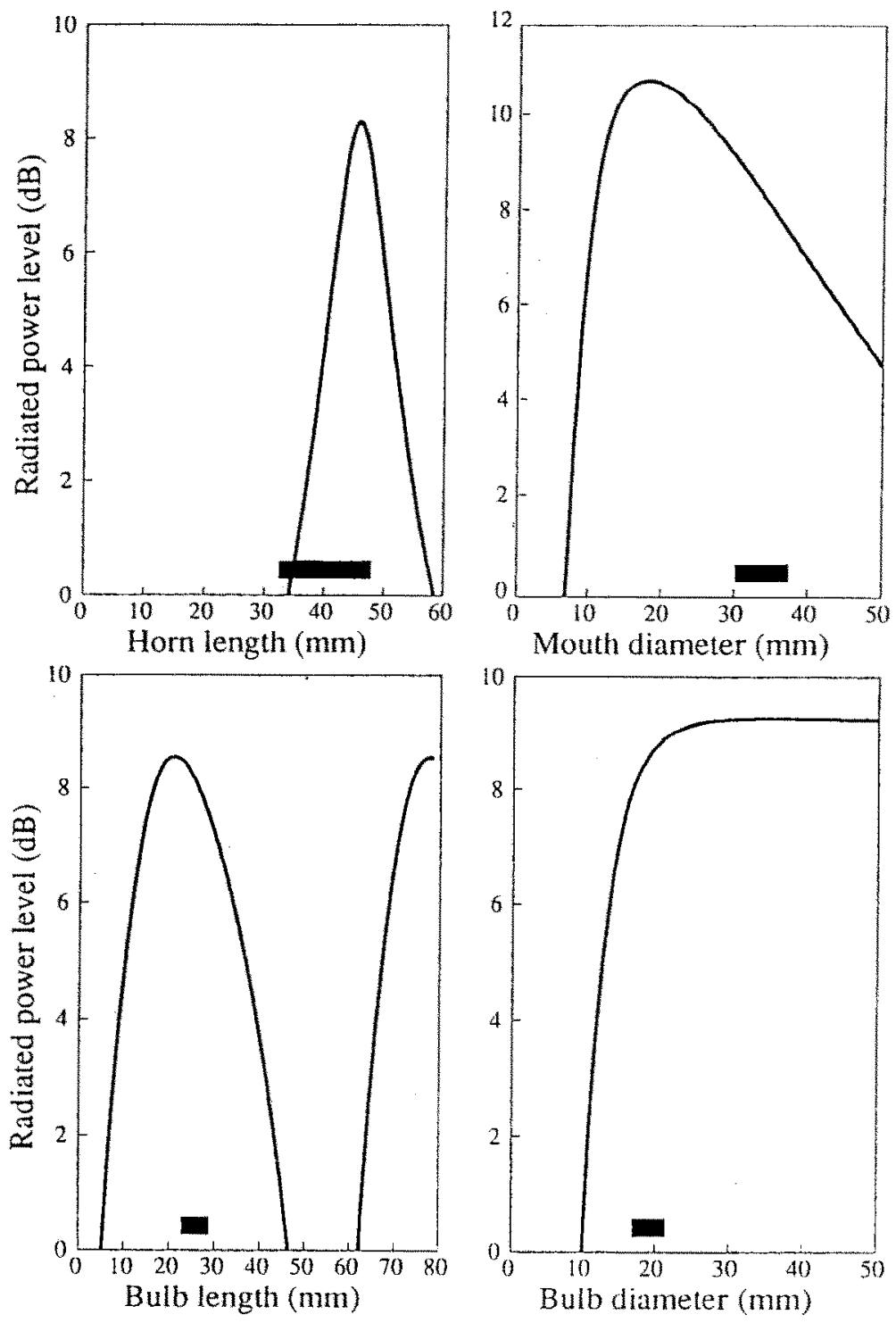

Figure 12. Calculated variation of the acoustic power level radiated from the burrow by a cricket (represented by the E.D.S.), singing at a frequency of $3 \mathrm{kHz}$, as the geometric parameters of the burrow are varied. The range of measured values \pm 1 s.d. is shown in each case as a solid bar. It can be seen that the actual burrow is close to optimal in terms of producing maximum power output at this frequency. If the singing frequency is $2.5 \mathrm{kHz}$, then all the curves should be expanded to the right by a factor 1.2 , thus increasing the optimal value of each dimension by this same factor. 
110

that $v=c$ as expected. If, however, the walls of the tube are porous, so that fluctuating air pressure extends for a characteristic exponentially attenuated distance $\delta$ into the walls, then the effective acoustic compliance $C$ must be calculated using this larger cross section $S^{\prime}=$ $\pi(a+\delta)^{2}$. The acoustic inertance $L$ is, however, unaffected since only the air in the free cross-section of the tube is able to move. The calculated wave speed is therefore not $c$ but rather the lower value

$$
v=c\left(\frac{a}{a+\delta}\right)
$$

This decrease of sound speed in the tube lowers the frequency of all the resonances by the same factor.

Replacing the normal value of sound speed $c$ by the reduced value $v$ in the calculations simply moves all the curves in Figures 10 and 11 downwards in frequency by a factor $v / c$. In the case of Figure 12, the curves and length scales are unaltered, but the assumed singing frequency is reduced by the same factor $v / c$. Most of the cricket burrow is between 10 and $20 \mathrm{~mm}$ in diameter, so that a penetration distance of about $1 \mathrm{~mm}$ into the porous soil would lower the effective sound speed by between 10 and 20 percent, bringing the measured and calculated results for the second burrow resonance into agreement with the singing frequency of the cricket.

In order to test this possibility we constructed two burrows of identical geometry using a former in the shape of a simple cylinder 80 $\mathrm{mm}$ long and $10 \mathrm{~mm}$ in diameter. In one case the burrow walls were of wax, and so were smooth as in our initial model, and in the other they were of loosely compacted soil. Each burrow was closed at one end and surrounded by a flat surface at its open end. The resonance frequencies of these two burrows were measured using an external source and a probe microphone. Two effects were immediately apparent. Firstly, the resonance peaks in the soil burrow were much lower and broader than in the wax burrow, an effect to be expected because of the viscous losses involved in pressure penetration into the porous soil. The decrease in resonance $Q$ was by about a factor of three. The second effect was a small but significant shift downwards of the resonance frequencies. The resonance near $3 \mathrm{kHz}$ shifted downwards by about $200 \mathrm{~Hz}$, which suggests a penetration depth of about $0.4 \mathrm{~mm}$ and reduces significantly the discrepancy between the measured and calculated resonances and the song frequency of the insect. Clearly this effect would depend quite significantly upon the porosity of the soil and the smoothness of the burrow walls, but the indication is that it may perhaps account for the frequency anomaly.

Another possibility is that our model for the cricket, as a sound source, is an inadequate representation of the real animal. In 
particular, we have assumed the vibrating wings to act as an ideal flow generator by-passed by a tube-like shunt, while at the resonance frequency of the wing panels the source impedance is really rather low. It has already been noted (Bennet-Clark 1970, 1987) that the cricket sound-generator is in fact a rather complex mechanism, but it is not clear that this should affect the optimal singing frequency.

\section{Comment on burrow resonances}

The model analysis confirms that the horn and cavity behave together like a long-necked resonator, the genuine "Helmholtz" (or Klipsch) resonance being at a frequency near $1200 \mathrm{~Hz}$, and the other resonances being higher modes of the system (Fletcher 1992, page 185). The resonance employed by the insect is the second mode, which has about three-quarters of a wavelength within the burrow, a pressure maximum near the rear of the bulb, and a pressure minimum and consequent $180^{\circ}$ phase change near the datum position in the constriction. The measurements in Figure 7 establish this unambiguously. We expect the phases in the horn and in the bulb to be nearly constant and to differ by $180^{\circ}$, though with some small dependence on position because of the component of the sound radiated from the horn at one end and absorbed in the burrow at the other. Calculation from the model confirms this different phase behaviour for the two resonances.

In an earlier publication (Bennet-Clark 1987) it was conjectured that the cricket constructed its burrow in such a way that it was able to use the lowest burrow resonance to enhance radiation of its song. This conjecture was supported by the observation that the dimensions of the burrow and cavity conformed approximately to those expected for the efficient short horn designed by Klipsch (1941). Our present analysis leads us to the conclusion that this agreement is fortuitous. The cavity volume is indeed such that the lowest resonance is placed just below the cut-off frequency of the horn as in the Klipsch design, as shown by the calculated pressure near the horn mouth in Figure 10 , but this resonance is at a frequency much below that of the insect song.

\section{CONCLUSION}

The results of the model calculations agree adequately well with measurements made on the artificial burrow. Clearly this statement is a matter of judgment, taking into account the approximations in the model, but no major features of the experimental results remain unaccounted for. This supports the conclusion that all the relevant physical factors have been included in the model and that the physical 
interpretation given in the main body of the present paper is correct. The one remaining puzzle is the small but significant discrepancy between the song frequency of the cricket and the resonance frequency of the burrow system. Indications are that this can be accounted for, at least in part, by porosity in the burrow walls, but further work is necessary to establish this unambiguously.

\section{APPENDIX}

Details of the calculation are important. In particular it is important to know the way in which the various impedances involved are evaluated in terms of the geometric dimensions of the horn, cavity and tunnel. For brevity we refer to definitions and equations in Fletcher (1992), denoted by a prefixed $F$, rather than giving extended information here.

The horn is specified in terms of its flare and dimensions by the acoustic impedance coefficients $H_{i j}$ which are identical to the coefficients $Z_{i j}$ defined by equations $\mathrm{F}(10.4)$ and $\mathrm{F}($ B.5)-(B.8). A similar discussion is given by Fletcher and Thwaites (1988). As far as this part of the calculation is concerned, it makes no difference whether we take the horn to be a single structure, as shown in Figure 9(a), or to consist of two identical horns, each having half of the total horn cross-section at each axial point, and effectively connected in parallel, in closer approximation to the real burrow.

The short pipe representing the bulb cavity is similarly specified by its coefficients $P_{i j}$ which are identical to the coefficients $Z_{i j}$ of equations $F(10.5)$ and $F(B .1)$. The exit burrow, treated as an infinite pipe, has acoustic impedance $Z_{\mathrm{B}}$ identical to $Z_{0}$ of $\mathrm{F}(10.3)$. The shunt around the cricket in Figure $9(\mathrm{~b})$ is taken to have a simple short-pipe impedance $Z_{\mathrm{s}}$ as specified for $Z_{\mathrm{N}}^{\mathrm{opn}}$ in $\mathrm{F}(10.6)$, with the area $S$ taken to be that of the space between the cricket and the walls of the constriction. In the case of external excitation, as in Figures $9(c)$ and 9 (d), the cricket is removed so that the cricket constriction becomes simply a short length of pipe. In specifying horn dimensions, we shall refer for convenience to the total horn length including the part in which the cricket sits, in all cases.

The radiation impedance at the open end of the burrow horn is denoted by $Z=R_{R}+j X_{R}$, where $R_{\mathrm{R}}$ is the radiation resistance contributed by the radiated sound wave, and $X_{\mathrm{R}}$ is the radiation reactance, which contributes the "end correction" at the open mouth of the horn. The analytical form of $Z_{\mathrm{R}}$ for an open circular pipe is given by equations $F(7.17)$ and its graphical behaviour in Figure $F(7.5)$. There is a correction to these equations because of the fact that the surface of the ground forms a flange around the horn mouth, but this is almost cancelled by an opposite correction for the fact that, as shown 
in Figure 1, the horn mouth actually consists of two apertures, rather than a single one.

The analog electrical network for the situation in which the cricket is singing is shown in Figure 13(a). The cricket, or the electrostatic dipole source, is represented by an acoustic flow generator of strength $U_{0}$, corresponding to the vibrating wings of the insect or

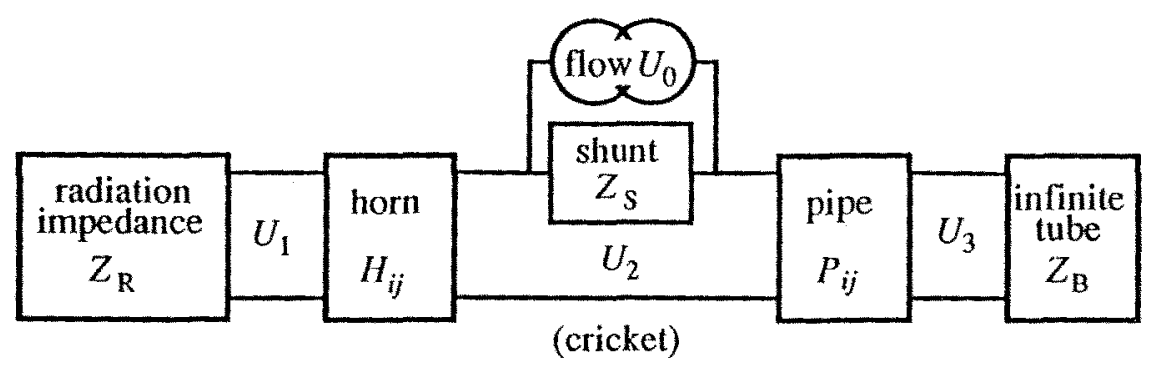

(a)

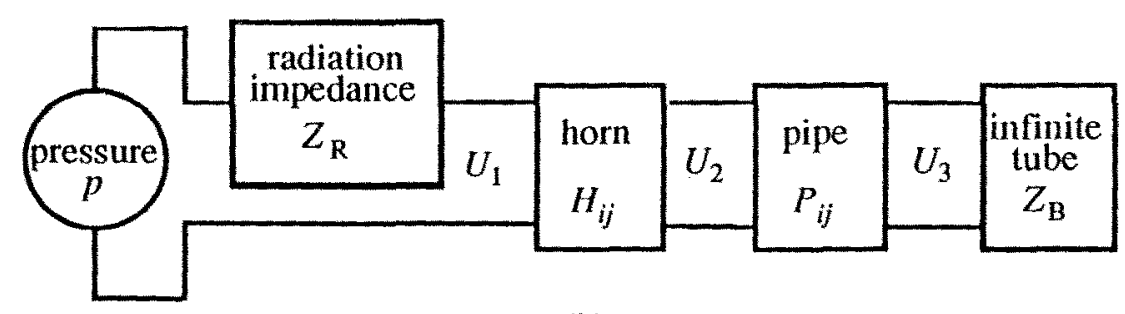

(b)

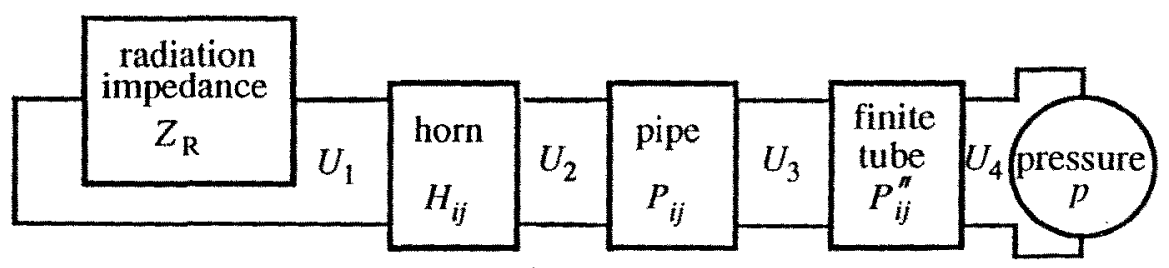

(c)

Figure 13. Electric analog networks for the mole-cricket burrow in the two configurations explored in the experiments: (a) with internal excitation by an E.D.S flow source or by the cricket's vibrating wings, (b) with external excitation from a plane wave incident on the burrow mouth, and (c) with external excitation from a source outside a truncated exit burrow. 
the diaphragm of the transducer. We justify this assumption in greater detail below. Similarly, when the burrow is excited by an external sound source, this is represented by a pressure generator of strength $p$ in the network of Figure 13(b). The values of $U_{0}$ and $p$ finally drop out of the calculation, since we are not calculating absolute levels but rather levels relative to the source in free air; only the source frequency is important.

As explained in detail by Fletcher and Thwaites (1979) and by Fletcher (1992, Chapters 10-11 and Appendix B), the behaviour of the networks of Figure 13 can be calculated by assuming a clockwise circulating acoustic current $U_{i}$ in each mesh of the network, as indicated by the symbols in the figure. We can then write down equations expressing the condition that the pressure drop around each mesh of the network is zero, unless there is a specific pressure source in that mesh (F, Section 8.8). For the internally-excited network of Figure 13(a), these equations are

$$
\begin{array}{r}
\left(Z_{\mathrm{R}}+H_{11}\right) U_{1}-H_{12} U_{2}=0 \\
-H_{12} U_{1}+\left(H_{22}+Z_{\mathrm{S}}+P_{11}\right) U_{2}-Z_{\mathrm{S}} U_{0}-P_{12} U_{3}=0 \\
-P_{12} U_{2}+\left(P_{22}+Z_{\mathrm{B}}\right) U_{3}=0
\end{array}
$$

These equations, in which the coefficients and variables are complex quantities, are very easily solved sequentially to give the acoustic currents $U_{i}$ in terms of the excitation current $U_{0}$ using a mixture of simple algebra and numerical evaluation. Calculation of a complete set of results over a large frequency range, together with evaluation of the other quantities of interest as outlined below, takes only a second or two on a standard desk-top computer.

The radiated sound power $\Pi$, which is the quantity of main interest, is the power dissipated in the real part $R_{R}$ of the radiation impedance $Z_{\mathrm{R}}$. This is

$$
\Pi=R_{\mathrm{R}} U_{1}^{2}
$$

if the flows $U_{i}$ are taken to be r.m.s. quantities, and is proportional to the square of the acoustic flow $U_{0}$ produced by the vibrating wings of the cricket, since $U_{1}$ is proportional to $U_{0}$.

Another quantity of interest is the sound pressure a small distance $d$ inside the mouth of the horn, given by

$$
p_{11}=\left(Z_{\mathrm{R}}+Z_{\mathrm{d}}\right) U_{1}
$$


where $Z_{\mathrm{d}}$ is the acoustic impedance of a short open tube of length $d$ and diameter equal to that of the horn mouth, as given by $F(10.6)$. Finally, we need the sound pressure in the centre of the bulb cavity, which is given by

$$
p_{\mathrm{C}}=\frac{1}{2} P_{12}^{\prime}\left(U_{2}-U_{3}\right)
$$

where $p_{12}^{r}$ is one of the impedance coefficients for a pipe of length equal to half that of the cavity, as given by equation $F(B .1)$.

Analysis of the externally-excited burrow of Figure 13(b) follows similar lines, the relevant equations being

$$
\begin{array}{r}
\left(Z_{12}+H_{11}\right) U_{1}-H_{12} U_{2}=p \\
-H_{12} U_{1}+\left(H_{22}+P_{11}\right) U_{2}-P_{12} U_{3}=0 \\
-P_{12} U_{2}+\left(P_{22}+Z_{B}\right) U_{3}=0
\end{array}
$$

The acoustic flows $U_{i}$ can again be evaluated, this time in terms of the excitation pressure $p$. The sound pressure $p_{\mathrm{C}}$ at the centre of the bulb can also be calculated from the expressions above. The sound pressure $p_{\mathrm{H}}$ just inside the horn is, however, now given by

$$
p_{11}=p-\left(Z_{\mathrm{R}}+Z_{\mathrm{h}}\right) U_{\mathrm{i}}
$$

and the pressure in the constriction is

$$
p_{\mathrm{X}}=P_{11} U_{2}-P_{12} U_{3}
$$

Again, all these quantities are proportional to the assumed value of the exciting pressure $p$.

Figure 13(c) shows the analog network for the experimental situation of Figure $9(\mathrm{~d})$. The network equations can be written down in the same way as for the case immediately above, but we shall not bother to do this because there is one additional loop to consider.

As an addendum we examine the internal impedance of the electret source and, by analogy, of the insect wings. Suppose the source diaphragm has area $S$, thickness $d$ and resonance angular frequency $\omega_{\mathrm{S}}$. If $\rho^{\prime} \approx 1000 \mathrm{~kg} \mathrm{~m}^{-3}$ is the density of the membrane material, then the acoustic inertance $L$ and acoustic compliance $C$ of the diaphragm are given by 


$$
\begin{gathered}
L=p^{\prime} d / S \\
C=S /\left(\rho^{\prime} d \omega_{S}^{2}\right)
\end{gathered}
$$

and the membrane impedance at frequency $\bar{\omega}$ is $R+j(L \sigma-1 / C \omega)$, where $R$ is the acoustic resistance, as determined by the $Q$ value of the transducer, which is probably about 2 .

In the case of the electret source, $d \approx 10 \mu \mathrm{m}$ and $\varpi_{\mathrm{S}} 10^{5} \mathrm{~s}^{-1}$, so that at the frequencies in our measurements the transducer impedance is at least $10^{7} \mathrm{SI}$ acoustic ohms, while the characteristic impedance of the burrow tubes is less than $3 \times 10^{6}$ ohms. The situation with the cricket is perhaps different, since the mode of wing excitation is different.

\section{ACKNOWLEDGEMENTS}

A.G.D. thanks the University of Melbourne for both the external study leave and an International Collaborative Research Grant, without which this research would not have been possible, and also the Department of Zoology, Oxford University for their kindness and hospitality. The authors are also grateful to Dr David Young and Dr Matthias Hennig for critically reading this manuscript and for their helpful comments.

\section{REFERENCE}

Bennet-Clark, H. C. (1970). The mechanism and efficiency of sound production in mole crickets. J. Exp. Biol., 52, 619-652.

Bennet-Clark, H. C. (1971). Acoustics of insect song. Nature, 234, 255-259.

Bennet-Clark, H. C. (1987). The tuned singing burrow of mole crickets. J. Exp. Biol., $128,383-409$

Bennet-Clark, H. C. and Young, D. (1992). A model of the mechanism of sound production in cicadas. J. Exp. Biol., 73, 123-153.

Daws, A. G. (1991). The use of resonance in the acoustic communication of Gryllotalpa australis. Hons. Thesis, The University of Melbourne. Unpublished.

Fletcher, N. H. (1992). Acoustic Systems in Biology. Oxford University Press; New York.

Fletcher, N. H. and Thwaites, S. (1979). Physical models for the analysis of acoustical systems in biology. Quart. Rev. Biophys., 12, 26-66 and 463.

Fletcher, N. H. and Thwaites, S. (1988). Obliquely truncated simple horns: Idealized models for vertebrate pinnae. Acustica, 65, 194-204.

Gough, W., Richards, J. P. G. \& Williams, R. P. (1983). Vibrations and Waves. Ellis Horwood Ltd.; Chichester.

Kavanagh, M, W. (1987). The efficiency of sound production in two cricket species, Gryllotalpa australis and Teleogryllus commodus (Orthoptera: Grylloidea). J. Exp. Biol, 130, 107-119.

Kavanagh, M. W. and Young, D. (1989). Bilateral symmetry of sound production in the mole cricket, Gryllotalpa australis. 2 . Comp. Physiol. A., 166, 43-49. 
Keefe, D. H. and Benade, A. H. (1983). Wave propagation in strongly curved ducts. $J$. Acoust. Soc. Am, 74, 320-332.

Klipsch, P. W. (1941). A low frequeney hom of small dimensions. d. Acoust. Soc. Amer., $13,137-144$.

Morse, P. M. (1948). Vibration and Sound. McGraw-Hill; New York (reprinted 1976 by the Acoustical Society of America, New York).

Nickerson, J. C., Snyder, D. E. and Oliver, C. C. (1979). Acoustical burrows construeted by mole crickets. Ann. Entomol. Soc. Amer., 72, 438-440.

Olson, H.F. (1957). Acoustical Engineering. Van Nostrand; Princeton.

Received 15 February 1996, revised 26 March 1996 and accepted 5 May 1996. 